
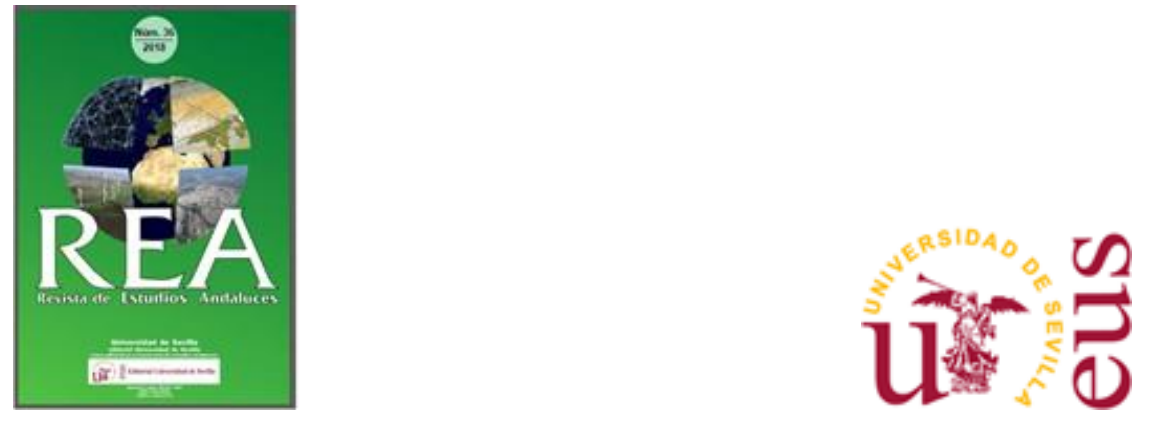

\author{
Revista de Estudios Andaluces (REA) \\ e-ISSN: 2340-2776.
}

REA Núm. 36 (2018). http://dx.doi.org/10.12795/rea.2018.i36

\title{
Discapacidad y Problemas de Salud como Barreras al Empleo en España
}

\section{Disability and Health Problems as Barriers to Employment in Spain}

\author{
María del Carmen Ponce-Caballero \\ Universidad de Huelva \\ maria.ponce.caballero@gmail.com \\ Leonel Caçador-Rodrigues \\ Universidad Internacional de Andalucía \\ leonel.rodrigues.uhu@gmail.com \\ Ana Millán-Tapia \\ Universidad Pablo de Olavide \\ amillan@upo.es \\ José María Millán-Tapia \\ Universidad de Huelva \\ jmillan@uhu.es
}

Formato de cita / Citation: Ponce-Caballero, Ma del Carmen, Caçador-Rodrigues, Leonel, MillánTapia, Ana y Millán-Tapia, José Mạ (2018). Discapacidad y Problemas de Salud como Barreras al Empleo en España. Revista de Estudios Andaluces, núm. 36, 178-207.

http://dx.doi.org/10.12795/rea.2018.i36.08

Enlace artículo/to link to this article: http://dx.doi.org/10.12795/rea.2018.i36.08 


\title{
Discapacidad y Problemas de Salud como Barreras al Empleo en España
}

\author{
Disability and Health Problems as Barriers to Employment in Spain
}

\author{
María del Carmen Ponce-Caballero \\ Universidad de Huelva \\ maria.ponce.caballero@gmail.com \\ Leonel Caçador-Rodrigues \\ Universidad Internacional de Andalucía \\ leonel.rodrigues.uhu@gmail.com \\ Ana Millán-Tapia \\ Universidad Pablo de Olavide \\ amillan@upo.es \\ José María Millán-Tapia \\ Universidad de Huelva \\ jmillan@uhu.es
}

Recibido: 12 de febrero, 2018

Revisado: 26 de junio, 2018

Aceptado: 27 de junio, 2018

\section{Resumen}

Este trabajo explora el papel que tienen diferentes tipos de limitaciones, enfermedades y problemas de salud sobre la probabilidad de trabajar y la probabilidad de pertenecer a distintos colectivos (empresarios, funcionarios, asalariados no funcionarios, desempleados, inactivos y estudiantes). Con este objetivo, y utilizando los microdatos de la Encuesta Europea de Salud en España de 2014, se estiman modelos de elección discreta con variable dependiente binaria y múltiple no ordenada. Nuestros principales resultados muestran cómo la probabilidad de tener un empleo es menor para aquellas personas con limitaciones, enfermedades y problemas de salud de tipo mental. Al distinguir entre colectivos, vemos cómo ni las limitaciones físicas ni las mentales afectan a la probabilidad de ser funcionario.

Palabras clave: discapacidad, limitaciones físicas y mentales, problemas de salud, mercado de trabajo, inclusión laboral.

\section{Abstract}

This paper explores the role of different types of limitations, diseases and health problems on both the probability of working and the probability of belonging to different groups (entrepreneurs, civil servants, other paid employees, unemployed, inactive individuals and students). To this end, we apply binary and multinomial non-ordered discrete choice models to microdata drawn from the Revista de Estudios Andaluces, núm. 36 (2018) pp. 178-207. e-ISSN: 2340-2776 http://dx.doi.org/10.12795/rea.2018.i36.08 
2014 European Health Interview Survey for Spain. Our main results show that the probability of having a job is lower for those individuals with mental limitations or health problems. When distinguishing between groups, we see that neither physical nor mental limitations affect the probability of being a civil servant.

Keywords: disability, physical and mental limitations, health problems, labour market, labour inclusion. 


\section{INTRODUCCIÓN}

La inclusión laboral de las personas con discapacidad es uno de los objetivos de la política sociolaboral en el que se viene trabajando intensamente desde hace años. En un trabajo reciente, Hernández y Millán (2015) expresaron su punto de vista en los siguientes términos:

La incorporación a un puesto de trabajo proporciona a la persona (con y sin discapacidad) un entorno fundamental de socialización y permite mayores niveles de independencia económica. Por tanto, garantizar el acceso al mercado laboral de las personas con discapacidad se presenta como requisito ineludible en el camino hacia una plena integración en la sociedad del colectivo. (Hernández y Millán 2015, pág. 30).

En este sentido, este colectivo se percibe cada vez menos como un grupo de personas con limitaciones físicas y sociales, y cada vez más como personas con objetivos y retos que pueden ser superados con el apoyo social e institucional adecuado (Livermore et al. 2000). En esta línea, el sector público promueve en España desde hace años una serie de medidas de importante calado que apoyan la inclusión laboral de las personas con discapacidad. Del mismo modo, las respectivas políticas de Responsabilidad Social Empresarial (RSE) de las empresas privadas también tratan de generar avances en esta materia (Cortés Ortiz 2011).

Sin embargo, los datos más recientes disponibles en España referidos a la situación de las personas con discapacidad en relación con el mercado de trabajo indican que los avances en este campo, pese a los indudables esfuerzos, son insuficientes. El cuadro a continuación muestra la evolución reciente de algunos datos agregados sobre la base de la estadística Empleo de las Personas con Discapacidad (EPD). ${ }^{1}$

Cuadro 1. Población de 16 a 64 años y relación con la actividad económica.

\begin{tabular}{|c|c|c|c|c|c|c|}
\hline & \multicolumn{3}{|c|}{ Personas sin discapacidad } & \multicolumn{3}{|c|}{ Personas con discapacidad } \\
\hline & 2014 & 2015 & 2016 & 2014 & 2015 & 2016 \\
\hline Tasa de actividad & 77,8 & 78,1 & 78,0 & 33,6 & 33,9 & 35,2 \\
\hline Tasa de empleo & 58,9 & 60,9 & 62,8 & 22,6 & 23,4 & 25,1 \\
\hline Tasa de paro & 24,3 & 21,9 & 19,5 & 32,7 & 31,0 & 28,6 \\
\hline
\end{tabular}

Fuente: EPD 2016.

Llama la atención en primer lugar cómo tan solo el 35,2\% de las 1.840 .700 personas con discapacidad reconocida en 2016 era activo, lo que representa una tasa de actividad 43 puntos por debajo de la correspondiente a la población sin discapacidad. Por su parte, la tasa de empleo de las personas con discapacidad fue del $25,1 \%$, con un aumento de 2,5 puntos respecto a 2014 , si bien todavía 40 puntos por debajo de la tasa para la población sin discapacidad. Finalmente, la tasa de desempleo se situó en el $28,6 \%$ para las personas con discapacidad, lo que supone una reducción

\footnotetext{
${ }^{1}$ La EPD surge a raíz de la firma en 2009 de un convenio de colaboración entre el Comité Español de Representantes de Personas con Discapacidad (CERMI), la Fundación ONCE, el Instituto de Mayores y Servicios Sociales (IMSERSO) y el Instituto Nacional de Estadística (INE), con la finalidad de obtener información sobre discapacidad y mercado laboral a través de la integración de registros administrativos con encuestas ya existentes.

Revista de Estudios Andaluces, núm. 36 (2018) pp. 178-207. e-ISSN: 2340-2776
}

http://dx.doi.org/10.12795/rea.2018.i36.08

Esta obra se distribuye con la licencia Creative Commons Reconocimiento-NoComercial-SinObraDerivada 4.0 Internacional (CC BY-NC-ND 4.0.) 
de más de 5 puntos con respecto a 2014, pero todavía 9 puntos por encima de la correspondiente a la población general.

A pesar de los avances tan limitados, el estudio de la discapacidad y su relación con la actividad económica en España se limita a un número reducido de trabajos, o bien de corte descriptivo, o bien de tipo condicional, sobre la base de datos relativamente obsoletos, y en los que predomina el análisis de la decisión de participación, sin distinguir entre los colectivos tan heterogéneos que coexisten dentro de cada una de estas dos situaciones: participación (ej. autoempleado, empleado asalariado, desempleado) o no participación (ej. estudiante, labores del hogar).

El principal objetivo de este trabajo es contribuir a la escasa literatura existente en este ámbito para España. En particular, nuestro objetivo es presentar, con los datos más recientes disponibles para España, un análisis condicional (o de regresión) sobre la actividad económica que permita contrastar el papel que tienen diferentes tipos de limitaciones, enfermedades y problemas de salud sobre, en primera instancia, la probabilidad de tener un empleo y, en una segunda fase, sobre la probabilidad de pertenecer a alguno de los siguientes colectivos: autoempleados, funcionarios, empleados asalariados -no funcionarios-, desempleados, personas inactivas - personas realizando labores del hogar y personas en situación de prejubilación- y estudiantes. Con este objetivo, y utilizando los microdatos de la Encuesta Europea de Salud en España de 2014, se estiman modelos de elección discreta con variable dependiente binaria y múltiple no ordenada. Nuestros principales resultados muestran cómo la probabilidad de tener un empleo es menor para aquellas personas con limitaciones, enfermedades y problemas de salud de tipo mental, lo que sugiere la necesidad de reforzar aquellas medidas activas y preventivas dirigidas a estos grupos con mayor riesgo de exclusión. Al distinguir entre colectivos, vemos cómo ni las limitaciones físicas ni las mentales afectan a la probabilidad de ser funcionario, lo que pone en valor el papel que está ejerciendo el estado en la inclusión laboral de las personas con discapacidad en España.

El resto del trabajo se organiza de la siguiente manera: la sección 2 se dedica a los antecedentes de este estudio; la sección 3 describe la base de datos, el modo en que se ha diseñado la muestra, las variables utilizadas en el análisis empírico y, de manera breve, el marco econométrico; la sección 4 aborda los resultados derivados del análisis empírico y, por último, la sección 5 presenta las conclusiones.

\section{ANTECEDENTES}

\subsection{DEFINICIÓN DE DISCAPACIDAD Y ANÁLISIS EMPÍRICO}

Una discapacidad hace referencia a la ausencia de la capacidad a la hora de realizar una actividad en la forma que se considera normal para un ser humano. Sin embargo, definir la discapacidad como discapacidad para trabajar genera un problema en el análisis del efecto de la discapacidad (para trabajar) sobre la participación laboral puesto que ambos elementos estarían conceptualmente unidos, lo que genera problemas evidentes de endogeneidad (Malo 2007). Sirvan como ejemplo las

Revista de Estudios Andaluces, núm. 36 (2018) pp.178-207. e-ISSN: 2340-2776 http://dx.doi.org/10.12795/rea.2018.i36.08

(c) (i) $\ominus$

(c) Esta obra se distribuye con la licencia Creative Commons Reconocimiento-NoComercial-SinObraDerivada 4.0 Internacional (CC BY-NC-ND 4.0.) 
preguntas que la encuesta estadounidense Health and Retirement Study (HRS) ${ }^{2}$ utiliza para recoger la información sobre discapacidad: ¿Tiene alguna deficiencia o problema de salud que limite la cantidad de trabajo remunerado que usted puede realizar? Si es así, ¿esa limitación le lleva en general a no trabajar? Además, este modo de formular las preguntas podría generar dos errores de medida potenciales: el sesgo de autojustificación y el sesgo de estigma (Malo 2007; Kreider y Pepper 2007, 2008). En el primero el individuo alega que es discapacitado para así justificar su no participación en el mercado de trabajo (overreporting), mientras que en el segundo alega que no es discapacitado para no sufrir el estigma de ser considerado por los demás como no apto para ciertas tareas o cometidos (underreporting).

Como alternativa, Malo (2007) sugiere que la definición de discapacidad utilizada (i) mida las limitaciones en las actividades de la vida diaria (que es el núcleo de las definiciones internacionales estandarizadas más recientes sobre discapacidad) ${ }^{3}$, (ii) no se base explícita y directamente en la discapacidad para trabajar y (iii) preferiblemente no sea una autoevaluación de conjunto sobre la salud. En otras palabras, para el análisis es más conveniente no utilizar información de los individuos que se auto-clasifican como persona con discapacidad, sino que a partir de una serie de preguntas y definiciones el investigador clasifica a los individuos como persona con o sin discapacidad. Sin embargo, tal y como apunta Malo (2007), el inconveniente principal de este tipo de definición es la necesidad de un cuestionario detallado y altamente especializado más propio de las encuestas de salud o discapacidad que de las generalistas o de aquellas con foco en la situación laboral. ${ }^{4}$ Una interesante excepción sería la pregunta sobre discapacidad que incluye la encuesta generalista European Union Statistics on Income and Living Conditions (EU-SILC) ${ }^{5}$ : ¿Se ha encontrado limitado en el desarrollo de su actividad diaria durante los últimos 6 meses (o cree que vaya a estarlo durante al menos 6 meses) por un problema de salud? Como vemos, si bien se trata de una autoevaluación de conjunto del propio individuo, se refiere a la actividad en general y no exclusivamente a la actividad laboral. ${ }^{6}$

\footnotetext{
${ }^{2}$ La HRS es una encuesta longitudinal de carácter anual llevada a cabo por la University of Michigan que se inicia en 1992 y que entrevista anualmente a unas 20.000 personas con edades cercanas a la jubilación. Por su propio diseño, esta encuesta se ha utilizado de forma extensiva para evaluar los efectos de la discapacidad en el comportamiento laboral de las personas mayores (ej. Bénitez-Silva 1999).

${ }^{3}$ La Clasificación Internacional del Funcionamiento, de la Discapacidad y de la Salud (CIF; en inglés International Classification of Functioning, Disability and Health -ICF) que emite la Organización Mundial de la Salud (OMS) define la discapacidad como un término genérico que abarca deficiencias, limitaciones de la actividad y restricciones a la participación. En concreto, la discapacidad es un fenómeno complejo que refleja una interacción entre las características del organismo humano (por ejemplo, una enfermedad como la parálisis cerebral, la depresión, etc.) y las características de la sociedad en la que vive (por ejemplo, actitudes negativas, transporte y edificios públicos inaccesibles, un apoyo social limitado, etc.).

${ }^{4}$ Para una revisión de cómo ha evolucionado y se ha incorporado a encuestas de salud el concepto de limitaciones en las actividades diarias, puede consultarse Gudex y Lafortune (2000).

${ }^{5}$ La EU-SILC, diseñada y coordinada por Eurostat, se inicia en 2004 para 12 países de la UE-15 y en la actualidad cubre más de 30 países europeos, incluyendo todos los de la UE-28. Su diseño como panel rotatorio anual, junto a la utilización del mismo cuestionario en todos los países, la convierte en una fuente de información armonizada y directamente comparable sobre los ingresos de los hogares, la distribución de la renta y la exclusión social. Sus características han permitido su uso en diferentes trabajos que analizan la relación entre discapacidad y mercado laboral (ej. Reeves et al. 2014).

${ }^{6}$ Para más información sobre distintas encuestas que incluyen información sobre discapacidad, véanse los trabajos de Gudex y Lafortune (2000) y Malo (2007).
}

Revista de Estudios Andaluces, núm. 36 (2018) pp. 178-207. e-ISSN: 2340-2776

http://dx.doi.org/10.12795/rea.2018.i36.08

(c) (†) $\ominus$

4.0 Internacional (CC BY-NC-ND 4.0.) 
Tal y como veremos con detalle en la subsección 3.3.2 sobre variables independientes principales, las definiciones sobre discapacidad y salud que utilizamos en este análisis sobre la base de los datos de la Encuesta Europea de Salud en España de 2014 se ajustan a los requisitos que sugiere Malo (2007) y son compatibles con las definiciones internacionales más recientes en tanto en cuanto hacen referencia a las limitaciones para realizar las actividades que la gente habitualmente hace.

\subsection{INTEGRACIÓN LABORAL DE LAS PERSONAS CON DISCAPACIDAD}

\subsubsection{BARRERAS A LA INTEGRACIÓN LABORAL}

En Livermore et al. (2000) se desarrolla una extensión de la teoría económica básica sobre la participación laboral para incorporar la situación de las personas con discapacidad (o problemas de salud). De este modo, entre los aspectos que pueden afectar a la participación laboral de este colectivo, han de tenerse en cuenta tanto aquellos que pueden afectar a la oferta como a la demanda de trabajo.

La oferta de trabajo se modeliza habitualmente para el individuo como un trade-off entre el trabajo que oferta y el "ocio" que demanda. En el caso de las personas con discapacidad, algunos atributos o condiciones hacen que el trabajo sea menos atractivo frente al "ocio". En primer lugar, las discapacidades "roban tiempo" a las personas que las padecen (cuidado médico y personal o incluso una menor esperanza de vida en determinados casos). Adicionalmente, participar en el mercado de trabajo puede generar costes adicionales a este colectivo (ej. rehabilitación, transporte especial y equipamiento, asistencia personal...).

Desde el lado de la demanda de trabajo, lo habitual es modelizarla a través de una decisión empresarial en la que la empresa compara la productividad de cada trabajador con el coste en que ha de incurrir para esta contratación. En el caso de este colectivo, la menor demanda de trabajo surge debido a que la empresa no solo percibe con frecuencia la discapacidad como una señal de baja productividad, sino que tiene en cuenta las eventuales necesidades de inversión para adaptar el puesto de trabajo a las condiciones específicas de estas personas.

Así, el papel del estado será fundamental a la hora de promover aumentos tanto en la oferta como en la demanda de trabajo de personas con discapacidad. La siguiente subsección presenta de manera sucinta las medidas de apoyo en esta materia.

\subsubsection{MEDIDAS DE APOYO A LA INTEGRACIÓN LABORAL EN ESPAÑA}

El sector público promueve en España desde hace años una serie de medidas de apoyo a la integración laboral de las personas con discapacidad. ${ }^{7}$ Estas medidas pueden agruparse en 3 grandes categorías:

\footnotetext{
${ }^{7}$ Rodríguez (2012a) y Hernández y Millán (2015) amplían esta información y analizan la efectividad de alguna de estas medidas.

Revista de Estudios Andaluces, núm. 36 (2018) pp.178-207. e-ISSN: 2340-2776 http://dx.doi.org/10.12795/rea.2018.i36.08

(c) $($ ) $\ominus$

cc) Esta obra se distribuye con la licencia Creative Commons Reconocimiento-NoComercial-SinObraDerivada 4.0 Internacional (CC BY-NC-ND 4.0.)
} 
1. Medidas destinadas a fomentar la integración en el sistema ordinario de trabajo. Dentro de estas medidas, destaca la cuota de reserva, que obliga a las empresas públicas y privadas con más de 50 trabajadores fijos a emplear a un número de trabajadores con discapacidad no inferior al $2 \%$. También se incluyen dentro de esta categoría una serie de subvenciones, incentivos, reducciones y bonificaciones de cuotas a la seguridad social que pretenden fomentar tanto la contratación por cuenta ajena como el empleo por cuenta propia en este colectivo.

2. Medidas destinadas a mejorar la integración en el sistema protegido de trabajo a través de los denominados Centros Especiales de Empleo (CEE).

3. Medidas para favorecer el tránsito desde el empleo protegido al ordinario a través de los enclaves laborales. Por enclave laboral se entiende un contrato entre una empresa del mercado ordinario de trabajo -empresa colaboradora-y un CEE para la realización de obras o servicios que guarden relación directa con la actividad principal de la empresa.

\subsection{REVISIÓN DE LA LITERATURA SOBRE DISCAPACIDAD Y EMPLEO}

\subsubsection{LITERATURA INTERNACIONAL}

La literatura económica sobre la relación entre discapacidad y mercado de trabajo ha abordado tres tópicos como ejes centrales: (i) la relación entre discapacidad y salarios (ej. Johnson y Lambrinos 1985; Baldwin y Johnson 2000; Kruse y Schur 2003); (ii) el impacto de la discapacidad sobre el empleo (ej. Parsons 1982; Yelin y Katz 1994; Gannon y Nolan 2003); y (iii) el efecto de los cambios legislativos (ej. DeLeire 2000; Acemoglu y Angrist 2001; Kruse y Schur 2003; Lalive et al. 2013). ${ }^{8}$ Dentro del segundo tópico, existen numerosos trabajos que analizan la relación entre discapacidad y participación laboral (ej. Stern 1989; Gannon 2005) y, en particular, esta misma relación para el caso de las personas con edades próximas a la jubilación (ej. Loprest et al. 1995; Riphahn 1999; Disney et al. 2006). El presente trabajo también se enmarca dentro de este segundo tópico, si bien analiza de manera específica la relación entre discapacidad y la probabilidad de tener un empleo. Dicho de otro modo, el outcome de interés no es tanto la pertenencia a la población activa como la propia ocupación de las personas con discapacidad (y problemas de salud).

Dentro de esta línea de trabajo, los estudios existentes sobre esta relación específica encuentran una relación negativa consistente entre discapacidad y la probabilidad de tener un empleo (ej. Baldwin y Johnson 1994, 1995; Blackaby et al. 1999; Jenkins y Rigg 2004; Madden 2004, Jones 2006). ${ }^{9}$ Más recientemente, algunos estudios han profundizado en esta relación examinando la relación entre discapacidad y el tipo de empleo. Se observa así como las personas con discapacidad muestran una mayor probabilidad de tener empleos tanto temporales (Schur 2003) como a tiempo

\footnotetext{
${ }^{8}$ Para una revisión de esta literatura, véase Jones (2008).

${ }^{9}$ Estos resultados son coherentes con los últimos datos agregados disponibles para la UE-28 provistos por Eurostat para el periodo 2011, en los que la tasa de empleo de las personas con dificultades para realizar alguna actividad básica (como ver, oír, caminar o comunicarse) fue del 47,3\%, casi 20 puntos porcentuales por debajo de la tasa de las personas sin tales dificultades. Al ampliar la definición de discapacidad a personas limitadas en el trabajo debido a un problema de salud prolongado y/o una dificultad para realizar alguna actividad básica, la tasa de empleo en la UE-28 baja al 38,1\%, situándose 30 puntos por debajo de la de aquellos que no declaran ninguna limitación en el trabajo.

Revista de Estudios Andaluces, núm. 36 (2018) pp. 178-207. e-ISSN: 2340-2776
}

http://dx.doi.org/10.12795/rea.2018.i36.08

Esta obra se distribuye con la licencia Creative Commons Reconocimiento-NoComercial-SinObraDerivada 4.0 Internacional (CC BY-NC-ND 4.0.) 
parcial (Schur 2003; Hotchkiss 2004), una mayor probabilidad de trabajar por cuenta propia (Blanck et al. 2000; Boylan y Burchardt 2002) y una mayor probabilidad de dedicarse a la realización de tareas en el hogar (Schur y Kruse 2002). Por otra parte, el modo en que el tipo y la severidad de la discapacidad influyen en la ocupación laboral también se ha explorado en la literatura. En este sentido, aquellos con enfermedades cardiovasculares, musculo-esqueléticas y respiratorias, aquellos que padecen enfermedades de tipo mental y aquellos que presentan múltiples limitaciones presentan menores opciones de ocupación que otras personas discapacitadas (Hum y Simpson 1996; Blackaby et al. 1999; Kidd et al. 2000; Zwerling et al. 2002; Wilkins 2004; Jones et al. 2006). La severidad de la discapacidad, independientemente de su tipo, también afecta negativamente a las tasas de empleo (Hale et al. 1998; Berthoud 2003; Jones 2011).

\subsubsection{LITERATURA NACIONAL}

En España, el estudio de la discapacidad y su relación con la actividad económica a través de las herramientas típicas de la economía laboral (esto es, el análisis condicional o de regresión) se limita a un número reducido de trabajos que analizan principalmente la participación laboral, esto es, la mera pertenencia a la población activa. ${ }^{10}$ Este es el caso del trabajo de Malo (2001), realizado con los microdatos para España del Panel de Hogares de la Unión Europea (PHOGUE) ${ }^{11}$ correspondientes al periodo 1994-95; los trabajos de Malo (2004), Malo y Pagán (2005), Dávila (2006), Dávila y Malo (2006) y Albarrán y Alonso (2010), todos ellos elaborados sobre la base de los microdatos de la Encuesta de Discapacidades, Deficiencias y Estado de Salud (EDDES-1999) ${ }^{12}$; y el trabajo de Cueto et al. (2012), realizado con los datos de la Encuesta de Discapacidad, Autonomía personal y situaciones de Dependencia (EDAD-2008). ${ }^{13}$ Este predominio es coherente con la baja tasa de actividad que se registra para este colectivo de forma sistemática en España. ${ }^{14}$

Sin embargo, la inclusión laboral de las personas con discapacidad pasa indefectiblemente por su pertenencia a la población ocupada. En este sentido, los trabajos que analizan esta relación para el caso español han sido mayoritariamente de corte descriptivo. Entre este tipo de trabajos se encuentran los realizados por Malo (2003), Villa-Fernández (2003), Giménez y Ramos (2003), Casado (2004), Pagán y Marchante (2004), Cazallas (2005), López y Seco (2005), Rodríguez-Cabrero et al.

\footnotetext{
${ }^{10}$ Para una revisión de la literatura sobre discapacidad y empleo en España, véase Rodríguez (2012b) y Ros-Clemente (2017).

${ }^{11}$ EI PHOGUE, diseñado y coordinado por Eurostat, es un panel fijo a nivel de la UE-15 que recoge información estadística sobre 76.500 hogares (equivalentes a unas 155.000 personas) durante el periodo 1994-2001. El mismo cuestionario ha sido utilizado en todos los países, lo que genera información armonizada y directamente comparable sobre diferentes aspectos del nivel y condiciones de vida y de la cohesión social.

12 La EDDES-1999 es una macroencuesta (89.000 hogares y 220.000 personas aproximadamente) elaborada por el INE que está orientada a cubrir las necesidades de información sobre los fenómenos de la discapacidad, la dependencia, el envejecimiento de la población y el estado de salud de la población residente en España.

${ }^{13}$ La EDAD-2008, también elaborada por el INE, es otra macroencuesta que sustituye a la EDDES-1999. Además de incorporar información más reciente, esta estadística aumenta su tamaño muestral ( 96.075 hogares, que equivalen a unas 260.000 personas), incorpora información sobre población institucionalizada (800 centros, que equivalen a unas 11.000 personas) y actualiza las definiciones de discapacidad a los estándares internacionales de la CIF.

${ }^{14}$ Para una revisión de las fuentes de datos que puedan ser utilizadas en el análisis de la relación entre discapacidad y empleo en España, véase Malo (2007) y Rodríguez (2013, 2017).
}

Revista de Estudios Andaluces, núm. 36 (2018) pp.178-207. e-ISSN: 2340-2776

http://dx.doi.org/10.12795/rea.2018.i36.08

(c) (i) $\ominus$

cc) Esta obra se distribuye con la licencia Creative Commons Reconocimiento-NoComercial-SinObraDerivada 4.0 Internacional (CC BY-NC-ND 4.0.) 
(2009), Jiménez y Huete (2010), Malo et al. (2011), Rodríguez (2012b, 2017), Rodríguez y Cueto (2013) y Hernández y Millán (2015). Hasta donde nuestro conocimiento alcanza, las únicas excepciones son los trabajos de García-Mainar (2000) y Cantanero y Moreno (2010), realizados respectivamente con los datos del PHOGUE 1994 para España y la EDAD-2008. Así, mediante modelos de elección discreta, García-Mainar analiza cómo el estado de salud afecta a la probabilidad de tener un empleo. Por su parte, utilizando la misma metodología, Cantanero y Moreno analizan la relación entre diferentes limitaciones y la probabilidad de tener un empleo temporal, tener un empleo a tiempo parcial, y trabajar por cuenta propia.

Resulta evidente, por tanto, la importancia de ampliar el número de análisis condicionales que estudien la relación entre discapacidad y pertenencia, no tanto a la población activa, sino a la población ocupada. Así mismo, se antoja crucial en este tipo de estudios tener en cuenta la heterogeneidad existente tanto en el conjunto de limitaciones o problemas de salud como en las actividades laborales existentes. Por último, contextualizar estos análisis a través del uso de datos más recientes también se revela como necesario. ${ }^{15}$

Cubrir estas carencias de la literatura existente en este ámbito para España es precisamente el principal objetivo de este trabajo, esto es, explorar con los datos más recientes disponibles para España el papel que tienen diferentes tipos de limitaciones, enfermedades y problemas de salud sobre, en primera instancia, la probabilidad de tener un empleo $y$, en una segunda fase, sobre la probabilidad de pertenecer a distintos colectivos (empresarios, funcionarios, asalariados no funcionarios, desempleados, inactivos y estudiantes).

\section{DATOS, DISEÑO MUESTRAL Y VARIABLES}

\subsection{FUENTE DE DATOS: ENCUESTA EUROPEA DE SALUD EN ESPAÑA 2014}

La Encuesta Europea de Salud (EES; en inglés European Health Interview Survey -EHIS), diseñada y coordinada por EUROSTAT, tiene como objetivo general proporcionar información sobre la salud de la población española de manera armonizada y comparable en el ámbito europeo con la finalidad de planificar y evaluar las actuaciones en materia sanitaria. Esta encuesta recoge información general sobre el hogar, además de información específica para cada miembro del hogar de 15 o más años sobre su estado de salud, enfermedades crónicas o de larga duración, calidad de vida relacionada con la salud, limitaciones para realizar las actividades de la vida diaria, acceso y utilización de los servicios sanitarios, consumo de medicamentos, prácticas preventivas, necesidades de atención médica no cubiertas, características físicas, consumo de tabaco y alcohol, ejercicio físico, alimentación, apoyo afectivo y personal y cuidados a otras personas.

La EES generó en su primera ola (2006-2009; en inglés EHIS wave 1 o EHIS round 2008) datos de sección cruzada (no longitudinales) para 18 países de la UE y Suiza: Austria y Estonia (2006); Eslovenia y Suiza (2007); Bélgica, Bulgaria, República Checa, Chipre, Francia, Letonia, Malta,

\footnotetext{
${ }^{15}$ Desde 2008 a 2014 podemos señalar que un evento importante para España ha sido la crisis económico-financiera o gran recesión que elevó el desempleo en 15 puntos porcentuales, del 9 al 24\%.

Revista de Estudios Andaluces, núm. 36 (2018) pp. 178-207. e-ISSN: 2340-2776

http://dx.doi.org/10.12795/rea.2018.i36.08
}

cc) (†) $\ominus$

4.0 Internacional (CC BY-NC-ND 4.0.) 
Rumanía, Turquía (2008); Alemania, Grecia, España, Hungría, Polonia, Eslovaquia (2009). La segunda ola (2013-2015, en inglés EHIS wave 2 o EHIS round 2014) ha ampliado su cobertura a todos los países de la UE-27 más Suiza, Islandia y Noruega, con una cobertura superior a los 210.000 hogares (e individuos). Sus microdatos ya están disponibles para algunos países como España, en lo que se denomina oficialmente la Encuesta Europea de Salud en España de 2014 (en adelante EESE 2014). El tamaño final de la muestra en esta encuesta es de 22.842 hogares (e individuos). Hasta donde nuestro conocimiento alcanza, este trabajo es el primero que explota estos datos para el análisis de la relación entre discapacidad y empleo.

\subsection{DISEÑO MUESTRAL}

A los individuos incluidos en la EESE 2014 (cuestionario de adultos) se les pregunta por el tipo de contrato o relación laboral que tienen, ofreciendo 9 posibles respuestas: funcionario, asalariado con contrato indefinido, asalariado con contrato temporal, asalariado con contrato verbal o sin contrato, empresario con asalariados, empresario sin asalariados, ayuda familiar, miembro de una cooperativa, u otras situaciones. Asimismo, se les pregunta (cuestionario de hogar) por la relación con la situación laboral actual, distinguiendo 7 situaciones: aquellos que tienen un empleo, desempleados, jubilados o prejubilados, estudiantes, incapacitados para trabajar, personas realizando labores del hogar e inactivos en otras situaciones.

En nuestra muestra de trabajo, excluimos tanto a aquellos individuos con edades fuera de la banda de 18-65 años (6.193 observaciones) como a aquellos pertenecientes a categorías poco relevantes para nuestro análisis y con un número de observaciones reducido. En particular, excluimos a los asalariados con contrato verbal o sin contrato, la ayuda familiar, los miembros de cooperativas, los que trabajan en otras situaciones, los incapacitados para trabajar y los inactivos en otras situaciones (808 observaciones).

Finalmente, después de excluir de nuestra muestra a aquellos individuos sin información en variables relevantes para la realización del análisis (838 observaciones), nuestra muestra final incluye 15.003 individuos agrupados en las siguientes actividades principales: empresario, funcionario, asalariado, desempleado, inactivo (labores del hogar y prejubilado) y estudiante.

\subsection{VARIABLES}

\subsubsection{VARIABLES DEPENDIENTES}

En este trabajo utilizamos la información sobre la relación laboral de los individuos para considerar dos variables dependientes. En primer lugar, la variable Tiene un empleo (0-1), de carácter discreto binario que toma el valor 1 para los individuos que tienen un empleo por cuenta propia o ajena y el valor 0 para los individuos que no tienen un empleo, ya sea como desempleados, inactivos (realizando labores del hogar o en situación de prejubilación) o estudiantes. En segundo lugar, la variable Actividad Principal (1-6), de carácter discreto y múltiple, tomando un valor diferente para cada una de las siguientes actividades: empresario, funcionario, asalariado, desempleado, inactivo

Revista de Estudios Andaluces, núm. 36 (2018) pp.178-207. e-ISSN: 2340-2776 http://dx.doi.org/10.12795/rea.2018.i36.08

(c) (i) $\ominus$

(c) Esta obra se distribuye con la licencia Creative Commons Reconocimiento-NoComercial-SinObraDerivada 4.0 Internacional (CC BY-NC-ND 4.0.) 
(labores del hogar y prejubilado) y estudiante. Se observa de este modo cómo el presente trabajo no solo contribuye a ampliar el número de análisis condicionales que estudian para España la relación entre discapacidad y pertenencia a la población empleada, sino que tiene en cuenta las diferentes opciones laborales existentes.

\subsubsection{VARIABLES INDEPENDIENTES}

\section{Discapacidad / estado de salud}

Incorporamos en nuestras estimaciones un conjunto de 6 variables ficticias (0-1) mutuamente excluyentes que capturan tanto alguna posible discapacidad como el estado de salud del individuo a través de los siguientes indicadores: $(a)$ limitación física, $(b)$ limitación mental -o física y mental-, $(c)$ enfermedad o problema de salud físico severo, $(d)$ enfermedad o problema de salud físico de gravedad intermedia o moderada, (e) enfermedad o problema de salud mental -o físico y mental-, y $(f)$ individuo física y mentalmente sano (que utilizamos como categoría de referencia). ${ }^{16}$ En este sentido, tener en cuenta en el análisis la heterogeneidad existente en el conjunto de limitaciones o problemas de salud es otra de las contribuciones de este trabajo.

En consonancia con la argumentación de Malo (2007) sobre los requisitos que han de cumplir las variables que capturen discapacidad y salud, nuestras variables asociadas a la existencia de limitaciones (indicadores $a, b$ ) no hacen referencia a limitaciones para trabajar, sino para "realizar las actividades que la gente habitualmente hace". Del mismo modo, las variables sobre el estado de salud (indicadores $c, d, e, f$ ) tampoco son una autoevaluación de conjunto sobre la salud, sino que hacen referencia a enfermedades y problemas de salud concretos.

\subsection{VARIABLES DE CONTROL}

\section{Variables demográficas}

Nuestras estimaciones incluyen el siguiente conjunto de variables demográficas: género (donde ser mujer es 1 y ser varón 0); edad y edad al cuadrado; estado civil, con 5 variables ficticias (0-1) (casado, separado, divorciado, viudo y soltero, siendo soltero la categoría de referencia); número de niños en el hogar; y nivel de estudios a través de 4 variables ficticias (0-1) (educación básica, secundaria, profesional y universitaria, siendo la primera categoría la que tomamos como referencia).

\section{Variables geográficas}

El siguiente conjunto de variables geográficas también se incluye en nuestro análisis: país de nacimiento (esta variable toma valor 1 si el individuo ha nacido en España y 0 si ha nacido en el extranjero); tamaño del municipio de residencia (variable discreta ordenada que toma valores entre 1 y 7 de menor a mayor tamaño del municipio); y comunidad autónoma de residencia, utilizando 19

\footnotetext{
${ }^{16}$ La descripción de cada una de estas categorías, así como del resto de variables utilizadas en el análisis, está disponible en el apéndice (Cuadro A1).

Revista de Estudios Andaluces, núm. 36 (2018) pp. 178-207. e-ISSN: 2340-2776
}

http://dx.doi.org/10.12795/rea.2018.i36.08

Esta obra se distribuye con la licencia Creative Commons Reconocimiento-NoComercial-SinObraDerivada 4.0 Internacional (CC BY-NC-ND 4.0.) 
variables ficticias (0-1) (17 CC.AA. además de las ciudades autónomas de Ceuta y Melilla, donde Andalucía será la comunidad de referencia).

\section{Variable económica}

Finalmente, incorporamos en nuestro análisis la siguiente variable económica: modalidad de seguro sanitario, que es una variable ficticia que toma valor 1 si el seguro sanitario del individuo es únicamente la sanidad pública (sin que el individuo esté asegurado a través de alguna de las mutualidades del estado) y valor 0 si el individuo utiliza otras modalidades de seguro sanitario incluyendo las mutualidades y seguros privados. ${ }^{17}$

\subsubsection{ANÁLISIS DESCRIPTIVO}

El Cuadro 2 a continuación muestra los estadísticos descriptivos de las variables consideradas en el análisis para cada una de las posibles situaciones laborales.

Como puede observarse, la menor proporción de individuos física y mentalmente sanos se encuentra en la categoría de inactivos $(19,7 \%)$ mientras que, por el contrario, la mayor proporción se encuentra en la categoría de estudiantes (62,9\%). Como consecuencia, la existencia de limitaciones (indicadores $a, b$ ) y problemas de salud (indicadores $c, e$ ) se produce en una mayor proporción en la población inactiva mientras que lo contrario ocurre para los estudiantes. Estas cifras son coherentes con la edad media de ambos grupos que, para el caso de los inactivos, alcanza los 56 años mientras que apenas alcanza los 22 años para los estudiantes. El resto de variables demográficas muestra cómo el perfil de la persona inactiva se corresponde con una mujer (74,3\%) casada $(73,3 \%)$ sin formación profesional ni universitaria $(80,6 \%)$. Por su parte, el perfil del estudiante es una persona soltera (98\%) con educación secundaria completada $(66,4 \%)$.

La población que no tiene un empleo se completa con el conjunto de individuos desempleados, cuya proporción de individuos física y mentalmente sanos (indicador $f ; 36,5 \%$ ) es la segunda más baja de la muestra. También este grupo presenta la segunda proporción más baja de individuos con enfermedades o problemas de salud físicos de gravedad intermedia o moderada (indicador $d ; 31 \%$ ). Por el contrario, este colectivo es el segundo que muestra tanto una mayor proporción de individuos con limitaciones físicas y mentales (indicadores $a, b$ ) como una mayor proporción de individuos con enfermedades o problemas de salud mental (indicador $e$ ). El perfil de la persona desempleada se corresponde con una persona de 42 años, o bien soltera $(35,8 \%)$ o bien casada $(52,4 \%)$, sin educación profesional o universitaria $(68,4 \%)$, y con escasos recursos económicos $(91,8 \%$ de la muestra asegurada únicamente a través de la sanidad pública).

\footnotetext{
17 Esta variable trata de capturar la situación económica del hogar. Asumimos aquí que aquellos individuos cuya modalidad de seguro médico sea únicamente la sanidad pública sin que el individuo esté asegurado a través de alguna de las mutualidades del estado, tendrán, en términos generales, una peor situación económica que aquellos individuos asegurados a través de la sanidad privada (mediante concierto individual o a través de la empresa en que trabajen) o en la sanidad pública o privada a través de estas mutualidades.
}

Revista de Estudios Andaluces, núm. 36 (2018) pp.178-207. e-ISSN: 2340-2776

http://dx.doi.org/10.12795/rea.2018.i36.08

(c) $($ ) $\ominus$

CC. Esta obra se distribuye con la licencia Creative Commons Reconocimiento-NoComercial-SinObraDerivada 4.0 Internacional (CC BY-NC-ND 4.0.) 
Cuadro 2. Estadísticos descriptivos.

\begin{tabular}{|c|c|c|c|c|c|c|}
\hline & \multicolumn{3}{|c|}{ Tiene un empleo } & \multicolumn{3}{|c|}{ No tiene un empleo } \\
\hline & Empresario & Funcionario & Asalariado & Desempleado & Inactivo & Estudiante \\
\hline Número de individuos & 1.752 & 1.160 & 6.278 & 2.908 & 2.191 & 714 \\
\hline$\%$ de la muestra & $11.7 \%$ & $7.7 \%$ & $41.8 \%$ & $19.4 \%$ & $14.6 \%$ & $4.8 \%$ \\
\hline Variables & $\begin{array}{c}\text { Media } \\
\text { (DS) }\end{array}$ & $\begin{array}{l}\text { Media } \\
\text { (DS) }\end{array}$ & $\begin{array}{l}\text { Media } \\
(D S)\end{array}$ & $\begin{array}{c}\text { Media } \\
\text { (DS) }\end{array}$ & $\begin{array}{c}\text { Media } \\
\text { (DS) }\end{array}$ & $\begin{array}{l}\text { Media } \\
\text { (DS) }\end{array}$ \\
\hline \multicolumn{7}{|l|}{ Estado de salud (6 variables ficticias) } \\
\hline a. Limitación física (0-1) & $14,0 \%$ & $14,7 \%$ & $12,8 \%$ & $17,5 \%$ & $26,1 \%$ & $7,56 \%$ \\
\hline b. Limitación mental -o fisica y mental- (0-1) & $1,20 \%$ & $1,55 \%$ & $1,21 \%$ & $3,23 \%$ & $4,15 \%$ & $1,26 \%$ \\
\hline c. Enfermedad o problema de salud físico severo (0-1) & $4,05 \%$ & $3,97 \%$ & $4,14 \%$ & $4,06 \%$ & $4,84 \%$ & $2,24 \%$ \\
\hline $\begin{array}{l}\text { d. Enfermedad o problema de salud físico de gravedad } \\
\text { intermedia o moderada (0-1) }\end{array}$ & $37,6 \%$ & $40,2 \%$ & $38,4 \%$ & $31,0 \%$ & $37,2 \%$ & $23,5 \%$ \\
\hline $\begin{array}{l}\text { e. Enfermedad o problema de salud mental } \\
\text {-o físico y mental- }(0-1)\end{array}$ & $4,05 \%$ & $3,44 \%$ & $3,64 \%$ & $7,64 \%$ & $7,99 \%$ & $2,52 \%$ \\
\hline$f$. Individuo física y mentalmente sano (0-1) (ref.) & $39,1 \%$ & $36,1 \%$ & $39,9 \%$ & $36,5 \%$ & $19,7 \%$ & $62,9 \%$ \\
\hline \multicolumn{7}{|l|}{ Variables demográficas } \\
\hline Mujer (0-1) & $34,4 \%$ & $56,3 \%$ & $47,8 \%$ & $53,0 \%$ & $74,3 \%$ & $53,9 \%$ \\
\hline Edad (18-65) & $\begin{array}{c}46,1 \\
(9,95)\end{array}$ & $\begin{array}{l}47,4 \\
(9,01)\end{array}$ & $\begin{array}{c}42,1 \\
(9,94)\end{array}$ & $\begin{array}{c}42,1 \\
(11,2)\end{array}$ & $\begin{array}{c}56,0 \\
(9.77)\end{array}$ & $\begin{array}{c}21,7 \\
(3,89)\end{array}$ \\
\hline \multicolumn{7}{|l|}{ Estado civil (5 variables ficticias) } \\
\hline Soltero (ref.) (0-1) & $24,0 \%$ & $24,4 \%$ & $29,9 \%$ & $35,8 \%$ & $8,58 \%$ & $98,0 \%$ \\
\hline Casado (0-1) & $65,6 \%$ & $62,0 \%$ & $59,7 \%$ & $52,4 \%$ & $73,3 \%$ & $1,54 \%$ \\
\hline Separado (0-1) & $5,71 \%$ & $7,41 \%$ & $6,01 \%$ & $6,50 \%$ & $2,56 \%$ & $0,14 \%$ \\
\hline Divorciado (0-1) & $1,60 \%$ & $2,76 \%$ & $1,70 \%$ & $1,62 \%$ & $4,66 \%$ & $0,28 \%$ \\
\hline Viudo (0-1) & $3,08 \%$ & $3,45 \%$ & $2,69 \%$ & $3,61 \%$ & $10,9 \%$ & $0 \%$ \\
\hline Número de niños en el hogar (0-6) & $\begin{array}{l}0,58 \\
(0,84)\end{array}$ & $\begin{array}{c}0,55 \\
(0,83)\end{array}$ & $\begin{array}{l}0,60 \\
(0,84)\end{array}$ & $\begin{array}{c}0,56 \\
(0,84)\end{array}$ & $\begin{array}{c}0,30 \\
(0,70)\end{array}$ & $\begin{array}{c}0,21 \\
(0,46)\end{array}$ \\
\hline \multicolumn{7}{|l|}{ Nivel de estudios (4 variables ficticias) } \\
\hline Educación básica (ref.) (0-1) & $15,1 \%$ & $1,64 \%$ & $11,0 \%$ & $24,7 \%$ & $45,4 \%$ & $2,80 \%$ \\
\hline Educación secundaria (0-1) & $40,8 \%$ & $21,4 \%$ & $38,0 \%$ & $43,7 \%$ & $35,2 \%$ & $66,4 \%$ \\
\hline Educación profesional (0-1) & $18,0 \%$ & $11,6 \%$ & $23,4 \%$ & $18,7 \%$ & $9,77 \%$ & $17,4 \%$ \\
\hline Educación universitaria (0-1) & $26,1 \%$ & $65,3 \%$ & $27,5 \%$ & $12,9 \%$ & $9,68 \%$ & $13,4 \%$ \\
\hline \multicolumn{7}{|l|}{ Variables geográficas } \\
\hline Nacido en España (0-1) & $92,0 \%$ & $98,3 \%$ & $88,1 \%$ & $85,1 \%$ & $91,8 \%$ & $90,1 \%$ \\
\hline Tamaño del municipio (1-7) & $\begin{array}{c}3,43 \\
(2,16)\end{array}$ & $\begin{array}{c}4,30 \\
(2,10)\end{array}$ & $\begin{array}{c}3,98 \\
(2,15)\end{array}$ & $\begin{array}{c}3,74 \\
(2,11)\end{array}$ & $\begin{array}{c}3,69 \\
(2,16)\end{array}$ & $\begin{array}{c}4,40 \\
(2,05)\end{array}$ \\
\hline \multicolumn{7}{|l|}{$\begin{array}{l}\text { Comunidad (o ciudad) autónoma } \\
\text { (19 variables ficticias) }\end{array}$} \\
\hline Andalucía (ref.) (0-1) & $10,3 \%$ & $9,48 \%$ & $9,40 \%$ & $17,1 \%$ & $13,7 \%$ & $12,6 \%$ \\
\hline Aragón (0-1) & $4,74 \%$ & $3,97 \%$ & $5,07 \%$ & $3,16 \%$ & $4,79 \%$ & $3,92 \%$ \\
\hline Asturias (0-1) & $4,05 \%$ & $5,09 \%$ & $3,09 \%$ & $3,03 \%$ & $4,66 \%$ & $4,90 \%$ \\
\hline Islas Baleares (0-1) & $4,17 \%$ & $4,22 \%$ & $4,46 \%$ & $2,99 \%$ & $2,88 \%$ & $3,64 \%$ \\
\hline Islas Canarias (0-1) & $4,00 \%$ & $3,45 \%$ & $4,97 \%$ & $6,84 \%$ & $5,02 \%$ & $4,62 \%$ \\
\hline Cantabria (0-1) & $4,11 \%$ & $2,93 \%$ & $3,35 \%$ & $2,58 \%$ & $5,66 \%$ & $2,24 \%$ \\
\hline Castilla y León (0-1) & $7,25 \%$ & $7,93 \%$ & $4,76 \%$ & $4,68 \%$ & $5,61 \%$ & $4,34 \%$ \\
\hline Castilla - La Mancha (0-1) & $5,19 \%$ & $5,17 \%$ & $3,98 \%$ & $5,81 \%$ & $5,89 \%$ & $4,62 \%$ \\
\hline Cataluña (0-1) & $10,7 \%$ & $6,72 \%$ & $12,0 \%$ & $7,94 \%$ & $8,81 \%$ & $8,96 \%$ \\
\hline Comunidad Valenciana (0-1) & $8,11 \%$ & $7,16 \%$ & $7,28 \%$ & $9,01 \%$ & $7,90 \%$ & $9,80 \%$ \\
\hline Extremadura (0-1) & $3,88 \%$ & $6,38 \%$ & $3,01 \%$ & $5,81 \%$ & $4,38 \%$ & $3,08 \%$ \\
\hline Galicia (0-1) & $7,13 \%$ & $5,60 \%$ & $3,97 \%$ & $5,36 \%$ & $5,75 \%$ & $5,74 \%$ \\
\hline Madrid (0-1) & $8,68 \%$ & $10,2 \%$ & $14,5 \%$ & $8,46 \%$ & $8,12 \%$ & $13,9 \%$ \\
\hline Murcia (0-1) & $4,62 \%$ & $4,83 \%$ & $4,36 \%$ & $4,99 \%$ & $4,38 \%$ & $5,18 \%$ \\
\hline Navarra (0-1) & $4,11 \%$ & $3,45 \%$ & $4,32 \%$ & $3,03 \%$ & $2,88 \%$ & $3,92 \%$ \\
\hline País Vasco (0-1) & $4,79 \%$ & $4,74 \%$ & $6,59 \%$ & $3,65 \%$ & $4,75 \%$ & $4,76 \%$ \\
\hline La Rioja (0-1) & $3,25 \%$ & $3,97 \%$ & $3,15 \%$ & $2,44 \%$ & $2,60 \%$ & $1,40 \%$ \\
\hline Ceuta $(0-1)$ & $0,34 \%$ & $2,07 \%$ & $0,88 \%$ & $0,76 \%$ & $0,96 \%$ & $1,12 \%$ \\
\hline
\end{tabular}

Revista de Estudios Andaluces, núm. 36 (2018) pp. 178-207. e-ISSN: 2340-2776

http://dx.doi.org/10.12795/rea.2018.i36.08

(c) (1) $\Theta$

cC Esta obra se distribuye con la licencia Creative Commons Reconocimiento-NoComercial-SinObraDerivada 4.0 Internacional (CC BY-NC-ND 4.0.) 


\begin{tabular}{|c|c|c|c|c|c|c|}
\hline Melilla (0-1) & $0,63 \%$ & $2,67 \%$ & $0,84 \%$ & $2,37 \%$ & $1,23 \%$ & $1,26 \%$ \\
\hline \multicolumn{7}{|l|}{ Variables económicas } \\
\hline Únicamente sanidad pública $(0-1)$ & $69,9 \%$ & $44,8 \%$ & $75,7 \%$ & $91,8 \%$ & $82,7 \%$ & $79,7 \%$ \\
\hline
\end{tabular}

Fuente: Elaboración propia a partir de los datos de la EESE 2014.

Por su parte, la población que tiene un empleo está clasificada en empresarios, funcionarios y trabajadores asalariados (no funcionarios). Tan solo el colectivo de estudiantes presenta una menor proporción de individuos con limitaciones y problemas de salud (indicadores $a, c, e$ ) que estos tres grupos. En particular, la proporción de individuos con limitaciones físicas (indicador $a$ ) de estos grupos oscila en torno al 14\%, mientras que tanto la proporción de individuos con enfermedades o problemas de salud físicos severos (indicador $c$ ) como la proporción de individuos con enfermedades o problemas de salud mental (indicador $e$ ) se sitúan en torno al $4 \%$. Los perfiles de estos grupos, sin embargo, presentan diferencias relevantes. El estereotipo de empresario se corresponde con un varón $(65,6 \%)$ casado $(65,6 \%)$ de 46 años con ciertos recursos económicos (el $30 \%$ de la muestra dispone de seguro médico privado). El estereotipo de asalariado no funcionario se corresponde con una persona casada $(59,7 \%)$ de 42 años con formación profesional o universitaria (50,9\%). Finalmente, el perfil del trabajador funcionario es una mujer (56,3\%) casada (62\%) de 47 años con educación universitaria (65,3\%).

\subsection{MARCO ECONOMÉTRICO}

Dada la naturaleza de nuestras variables dependientes se han utilizado diferentes métodos de estimación. Así, cuando la variable dependiente analizada es Tener un empleo (0-1), utilizamos modelos de elección discreta con variable dependiente binaria, en particular, modelos logit binomiales. Cuando por el contrario la variable dependiente analizada es la Actividad Principal (16), utilizamos modelos de elección discreta con variable dependiente múltiple no-ordenada, en particular, modelos logit multinomiales.

\subsection{PRINCIPALES RESULTADOS}

En esta sección comentamos los resultados relativos a las variables que captan el estado de salud del individuo, centrándonos en primer lugar en la especificación 1 en la que analizamos la probabilidad de tener un empleo (frente a la alternativa de no tenerlo). En este sentido, podemos observar cómo las limitaciones para "realizar las actividades que la gente habitualmente hace", ya sean físicas o mentales (indicadores $a, b$ ), disminuyen la probabilidad de tener un empleo. Estos resultados son coherentes con aquellos que observan una relación negativa entre discapacidad y la probabilidad de tener un empleo (ej. Baldwin y Johnson 1994, 1995; Blackaby et al. 1999; GarcíaMainar 2000); Jenkins y Rigg 2004; Madden 2004; Jones 2006). En particular, las limitaciones físicas (indicador $a$ ) disminuyen esta probabilidad un $10,8 \%$ mientras que las mentales (indicador $b$ ) lo hacen en un $30,4 \%$.

En cuanto a las enfermedades o problemas de salud (indicadores $c, d, e$ ), en el caso de que estos sean de tipo físico y de carácter severo (indicador $c$ ), la probabilidad de tener un empleo disminuye

Revista de Estudios Andaluces, núm. 36 (2018) pp.178-207. e-ISSN: 2340-2776

http://dx.doi.org/10.12795/rea.2018.i36.08

(c) (†) $\ominus$

cc) Esta obra se distribuye con la licencia Creative Commons Reconocimiento-NoComercial-SinObraDerivada 4.0 Internacional (CC BY-NC-ND 4.0.) 
un 4,4\% (siendo este resultado tan solo significativo de forma marginal), mientras que si son de tipo mental (indicador $e$ ) esta probabilidad baja un $17,6 \%$. Estos resultados también dan soporte a lo obtenido en otros trabajos que identifican cómo los problemas de salud mental tienen una influencia más negativa en el empleo (ej. Blackaby et al. 1999; Kidd et al. 2000; Jones et al. 2006). Finalmente, observamos cómo aquellos que presentan alguna enfermedad o problema de salud físico de gravedad intermedia o moderada (indicador $d$ ) ven cómo su probabilidad de tener un empleo sube en torno a un 3\%. En este sentido, dos comentarios emergen a la luz de este resultado.

En primer lugar, este resultado junto con el obtenido para las enfermedades o problemas de salud de tipo físico y de carácter severo (indicador c) parece confirmar cómo la severidad de la discapacidad también afecta negativamente a las tasas de empleo (Hale et al. 1998; Berthoud 2003; Jones, 2011). En segundo lugar, el signo positivo del coeficiente asociado a este indicador podría parecer anómalo. Sin embargo, es importante aclarar que, frente a otros indicadores, esta categoría agrupa los problemas de salud más leves y por tanto menos influyentes a priori en las opciones laborales de los individuos. Además, este grupo de personas presenta una edad media 6 años superior al que presenta el colectivo de personas física y mentalmente sanas, lo que debe contribuir positivamente a sus opciones de encontrar un empleo.

La especificación 2, esto es, aquella que nos permite distinguir entre 6 actividades principales, arroja también resultados interesantes para las variables sobre limitaciones y problemas de salud (indicadores $a-e$ ). En líneas generales, y en consonancia con lo obtenido en la especificación 1, vemos cómo para aquellas actividades que supongan tener un empleo (empresario, funcionario y asalariado), al menos uno de los coeficientes asociados a estos indicadores es negativo y significativo. Del mismo modo, para aquellas actividades que supongan no tener un empleo (desempleado, inactividad y estudiante), al menos uno de estos coeficientes indicados es positivo y significativo.

En particular, vemos cómo la probabilidad de ser empresario con o sin asalariados disminuye un $17,6 \%$ para los individuos con limitaciones de tipo físico (indicador $a$ ) y un $36,9 \%$ para aquellos que sufren limitaciones mentales (indicador $b$ ). Estos resultados no son consistentes con los obtenidos por Blanck et al. 2000 y Boylan y Burchardt 2002 para el Reino Unido, que observan cómo las personas con discapacidad muestran una mayor probabilidad de trabajar por cuenta propia. En relación con las enfermedades o problemas de salud de tipo físico, vemos como si son de gravedad severa (indicador $c$ ) disminuyen las opciones de ser autoempleado un $22,7 \%$, mientras que si son de gravedad intermedia o moderada (indicador $d$ ) no parecen tener efectos sobre la probabilidad de pertenecer a este grupo. Tampoco las enfermedades o problemas de salud de tipo mental (ej. ansiedad, depresión; indicador e) parecen afectar a la probabilidad de ser empresario.

La probabilidad de tener un empleo como funcionario del estado, por su parte, no parece estar afectada por ningún tipo de limitación de tipo físico ni mental (indicadores $a, b$ ). Las enfermedades o problemas de salud de tipo físico tampoco parecen afectar a las opciones de ser funcionario (indicadores $c, d$ ). Tan solo en el caso de que las enfermedades o problemas de salud sean de tipo mental (ej. ansiedad, depresión; indicador e) la probabilidad de ser funcionario cae, haciéndolo un 
Cuadro 3. Determinantes de la actividad principal-Modelos logit binomial y multinomial-.

Especificación

Modelo

Variable dependiente $(y)$ Logit binomial

Probabilidades predichas $(\hat{\mathrm{y}})$

Tener un empleo (0-1) Acividad Principal

\section{Variables independientes $(x)$}

\begin{tabular}{ccccccccccccccc}
\multicolumn{2}{c}{0,613} & \multicolumn{2}{c}{0,117} & \multicolumn{2}{c}{0,077} & \multicolumn{2}{c}{0,418} & \multicolumn{2}{c}{0,194} & \multicolumn{2}{c}{0,048} & \multicolumn{2}{c}{0,146} \\
$\frac{d y}{y} \%$ & $\mathrm{z}$ & $\frac{d y / d x}{y} \%$ & $\mathrm{z}$ & $\frac{d y / d x}{y} \%$ & $\mathrm{z}$ & $\frac{d y / d x}{y} \%$ & $\mathrm{z}$ & $\frac{d y / d x}{y} \%$ & $\mathrm{z}$ & $\frac{d y / d x}{y} \%$ & $\mathrm{z}$ & $\frac{d y / d x}{y} \%$ & $\mathrm{z}$ \\
\hline
\end{tabular}

salariado

Desempleado

Inactivo

\begin{tabular}{ccccccccccccccc}
\hline & 0,613 & \multicolumn{2}{c}{0,117} & \multicolumn{2}{c}{0,077} & \multicolumn{2}{c}{0,418} & \multicolumn{2}{c}{0,194} & \multicolumn{2}{c}{0,048} & 0,146 \\
$\frac{d y / d x}{y} \%$ & $\mathrm{z}$ & $\frac{d y / d x}{y} \%$ & $\mathrm{z}$ & $\frac{d y / d x}{y} \%$ & $\mathrm{z}$ & $\frac{d y / d x}{y} \%$ & $\mathrm{z}$ & $\frac{d y / d x}{y} \%$ & $\mathrm{z}$ & $\frac{d y / d x}{y} \%$ & $\mathrm{z}$ & $\frac{d y / d x}{y} \%$ & $\mathrm{z}$ \\
\hline
\end{tabular}

Estudiante

Estado de salud (6 variables ficticias)

a. Limitación física (0-1)

b. Limitación mental -o física y mental- (0-1)

c. Enfermedad o problema de salud físico severo (0-1)

d. Enfermedad o problema de salud físico de gravedad

intermedia o moderada (0-1)

e. Enfermedad o problema de salud mental

-o físico y mental- (0-1)

$f$. Individuo física y mentalmente sano (0-1) (ref.)

Variables demográficas

\section{Mujer (0-1)}

Edad (18-65)

Edad al cuadrado

Estado civil (5 variables ficticias)

Soltero (ref.) (0-1)

Casado (0-1)

Separado (0-1)

Divorciado $(0-1)$

Viudo (0-1)

Número de niños en el hogar (0-6)

Nivel de estudios (4 variables ficticias)

Educación básica (ref.) (0-1)

Educación secundaria (0-1)

Educación profesional (0-1)

Educación universitaria (0-1)

$\begin{array}{rlllllllllllll}-10,8 & -5,97 * * & -17,6 & -2,61 * * * & -3,66 & -0,45 & -10,7 & -3,80^{* * *} & 24,9 & 4,83 * * * & 13,9 & 2,84 * * * & -1,45 & -0,16 \\ -30,4 & -7,02 * * & -36,9 & -2,46^{* *} & -10,4 & -0,54 & -33,1 & -5,24 * * & 56,3 & 4,45 * * & 42,8 & 3,62 * * * & 37,5 & 1,65 * \\ -4,37 & -1,45 & -22,7 & -2,20^{* *} & -12,3 & -0,95 & 0,89 & 0,19 & 10,8 & 1,27 & 10,2 & 1,25 & -7,51 & -0,49 \\ 3,14 & 2,29 * * & -7,29 & -1,36 & -1,83 & -0,30 & 6,10 & 2,83 * * * & -3,80 & -1,03 & -3,61 & -0,91 & -6,18 & -1,10 \\ & & & & & & & & & & & & & \\ -17,6 & -6,23 * * * & -14,0 & -1,27 & -24,3 & -2,02 * * & -18,0 & -4,10^{* * *} & 53,5 & 6,30 * * & 6,04 & 0,86 & -4,23 & -0,28\end{array}$

Variables geográficas

Nacido en España (0-1)

Tamaño del municipio (1-7)

$\begin{array}{rrrrrlrlllllll}-21,9 & -18,8^{* * *} & -66,4 & -14,6^{* * *} & 9,62 & 1,84^{*} & -15,8 & -8,57 * * & 6,00 & 1,84^{*} & 82,0 & 25,8^{* * *} & 10,2 & 2,12 * * \\ 12,1 & 39,0^{* * *} & 7,65 & 4,41^{* * *} & 17,3 & 6,94^{* * *} & 6,23 & 9,48^{* * *} & 1,15 & 1,15 & -23,5 & -21,1^{* * *} & -34,2 & -18,7 * *\end{array}$

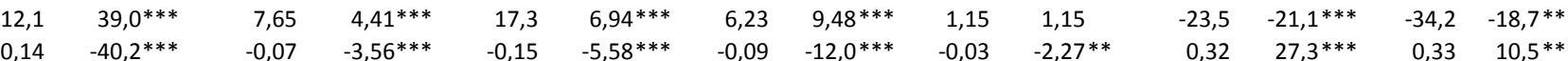

Comunidad (o ciudad) autónoma (19 variables ficticias)

\begin{tabular}{|c|c|c|c|c|c|c|c|c|c|c|c|c|}
\hline $3,32 * * *$ & 1,34 & 0,22 & $-16,7$ & $-2,27^{* *}$ & 9,27 & $3,66 * * *$ & $-35,2$ & $-7,39 * * *$ & 50,1 & $11,2^{* * *}$ & $-68,2$ & $-7,52^{* * *}$ \\
\hline $2,87^{* * *}$ & $-0,38$ & $-0,03$ & $-3,19$ & $-0,19$ & 9,18 & 1,48 & $-10,4$ & $-0,95$ & 1,34 & 0,17 & $-36,5$ & $-0,97$ \\
\hline $3,00^{* * *}$ & $-2,49$ & $-0,24$ & $-6,04$ & $-0,51$ & 8,02 & $1,71^{*}$ & $-15,3$ & $-1,84 *$ & 5,94 & 0,92 & $-10,3$ & $-0,32$ \\
\hline 0,62 & $-18,0$ & $-1,13$ & 13,1 & 0,64 & 21,8 & $3,15^{* * *}$ & $-58,9$ & $-5,71 * * *$ & 58,0 & $6,59 * * *$ & $-107,0$ & $-29,5^{* * *}$ \\
\hline$-4,64 * * *$ & 1,97 & 0,63 & $-4,34$ & $-1,17$ & $-6,70$ & $-5,21^{* * *}$ & 0,63 & 0,28 & 23,1 & $9,59 * * *$ & $-12,3$ & $-2,43^{* *}$ \\
\hline $7,99 * * *$ & 9,18 & 1,35 & 50,4 & $11,5^{* * *}$ & 7,11 & $2,42 * *$ & $-44,3$ & $-7,85 * * *$ & $-18,1$ & $-3,75 * * *$ & 69,0 & $8,97^{* * *}$ \\
\hline $11,8^{* * *}$ & 1,86 & 0,24 & 60,2 & $9,56 * * *$ & 21,4 & $6,29 * * *$ & $-56,1$ & $-8,83^{* * *}$ & $-39,0$ & $-6,62^{* * *}$ & 57,8 & $6,74^{* * *}$ \\
\hline $20,7 * * *$ & 15,6 & $1,94^{*}$ & 218,3 & $25,0 * * *$ & 12,4 & $3,74 * * *$ & $-91,4$ & $-15,2 * * *$ & $-66,1$ & $-12,3^{* * *}$ & 72,9 & $7,93 * * *$ \\
\hline
\end{tabular}

Andalucía (ref.) (0-1)

Revista de Estudios Andaluces, núm. 36 (2018) pp. 178-207. e-ISSN: 2340-2776

http://dx.doi.org/10.12795/rea.2018.i36.08

\section{cc) (i) $(\Theta$}




\begin{tabular}{|c|c|c|c|c|c|c|c|c|c|c|c|c|c|c|}
\hline Aragón (0-1) & 20,3 & $6,35 * * *$ & 13,9 & 1,13 & $-17,5$ & $-1,28$ & 29,6 & $5,82 * * *$ & $-54,4$ & $-6,03 * * *$ & $-10,1$ & $-1,16$ & $-13,6$ & $-1,10$ \\
\hline Asturias (0-1) & 15,2 & $4,47^{* * *}$ & 27,2 & $1,93 *$ & 35,6 & $2,06 * *$ & 8,14 & 1,50 & $-51,7$ & $-5,55 * * *$ & $-1,88$ & $-0,20$ & 20,2 & 1,48 \\
\hline Islas Baleares (0-1) & 24,3 & $7,32^{* * *}$ & 12,0 & 0,94 & 1,73 & 0,11 & 34,3 & $6,35^{* * *}$ & $-49,9$ & $-5,21 * * *$ & $-42,5$ & $-5,03 * * *$ & $-0,49$ & $-0,04$ \\
\hline Islas Canarias (0-1) & 9,12 & $2,99 * * *$ & $-5,14$ & $-0,45$ & $-15,9$ & $-1,11$ & 17,9 & $3,72 * * *$ & $-20,3$ & $-2,32 * *$ & $-13,7$ & $-1,65 *$ & 6,09 & 0,51 \\
\hline Cantabria (0-1) & 15,4 & $4,50 * * *$ & 25,4 & $1,83 *$ & $-5,36$ & $-0,33$ & 17,8 & $3,23 * * *$ & $-64,4$ & $-7,19 * * *$ & 20,5 & $2,05 * *$ & $-10,7$ & $-0,72$ \\
\hline Castilla y León (0-1) & 18,5 & $6,26 * * *$ & 37,9 & $3,15^{* * *}$ & 42,4 & $2,83 * * *$ & 8,14 & $1,75^{*}$ & $-47,9$ & $-5,79 * * *$ & $-18,2$ & $-2,31 * *$ & 17,6 & 1,35 \\
\hline Castilla - La Mancha (0-1) & 7,44 & $2,40 * *$ & 11,9 & 1,02 & 51,5 & $2,89 * * *$ & $-0,90$ & $-0,19$ & $-32,1$ & $-3,75^{* * *}$ & 6,22 & 0,70 & 6,53 & 0,54 \\
\hline Cataluña (0-1) & 19,3 & $7,72^{* * *}$ & 10,5 & 1,13 & $-43,9$ & $-4,32 * * *$ & 34,8 & $8,93 * * *$ & $-50,6$ & $-7,12 * * *$ & $-19,8$ & $-2,90 * * *$ & 6,38 & 0,64 \\
\hline Comunidad Valenciana (0-1) & 8,27 & $3,10^{* * *}$ & 13,4 & 1,32 & 5,84 & 0,45 & 8,35 & $2,04 * *$ & $-24,6$ & $-3,22 * * *$ & $-16,5$ & $-2,29 * *$ & 35,3 & $3,31 * * *$ \\
\hline Extremadura (0-1) & 4,63 & 1,39 & $-4,30$ & $-0,37$ & 89,0 & $4,59 * * *$ & $-6,68$ & $-1,33$ & $-6,85$ & $-0,71$ & $-13,1$ & $-1,49$ & $-7,26$ & $-0,54$ \\
\hline Galicia (0-1) & 12,0 & $3,95 * * *$ & 47,8 & $3,78^{* * *}$ & 28,7 & $1,82 *$ & $-0,24$ & $-0,05$ & $-33,5$ & $-3,88 * * *$ & $-23,1$ & $-2,94 * * *$ & 45,3 & $3,41 * * *$ \\
\hline Madrid (0-1) & 20,4 & $8,22 * * *$ & $-9,09$ & $-1,01$ & $-39,7$ & $-4,03 * * *$ & 42,0 & $10,9 * * *$ & $-49,5$ & $-6,94 * * *$ & $-30,4$ & $-4,53^{* * *}$ & 12,7 & 1,38 \\
\hline Murcia (0-1) & 11,6 & $3,68 * * *$ & 16,6 & 1,33 & 20,6 & 1,29 & 10,2 & $2,07^{* *}$ & $-32,9$ & $-3,71 * * *$ & $-11,1$ & $-1,28$ & 4,12 & 0,34 \\
\hline Navarra (0-1) & 23,7 & $7,12 * * *$ & 11,6 & 0,90 & $-0,52$ & $-0,03$ & 32,7 & $6,04^{* * *}$ & $-55,2$ & $-6,03 * * *$ & $-32,4$ & $-3,69 * * *$ & 9,02 & 0,63 \\
\hline País Vasco (0-1) & 19,9 & $6,58^{* * *}$ & $-5,82$ & $-0,54$ & $-28,2$ & $-2,34 * *$ & 38,1 & $8,06 * * *$ & $-58,0$ & $-6,92 * * *$ & $-17,8$ & $-2,15^{* *}$ & 15,5 & 1,21 \\
\hline La Rioja (0-1) & 20,1 & $5,44 * * *$ & 18,0 & 1,24 & 40,3 & $2,11 * *$ & 17,6 & $3,02 * * *$ & $-56,6$ & $-5,79 * * *$ & $-5,63$ & $-0,52$ & $-16,8$ & $-1,03$ \\
\hline Ceuta (0-1) & 14,3 & $2,29 * *$ & $-45,9$ & $-2,48 * *$ & 131,6 & $3,60^{* * *}$ & 6,70 & 0,72 & $-56,3$ & $-3,53 * * *$ & 20,1 & 1,05 & 7,68 & 0,35 \\
\hline Melilla (0-1) & $-0,13$ & $-0,02$ & $-29,7$ & $-1,61$ & 138,2 & $4,03 * * *$ & $-18,2$ & $-2,34 * *$ & 21,7 & 1,31 & $-20,9$ & $-1,54$ & $-15,8$ & $-0,85$ \\
\hline Variable económica & & & & & & & & & & & & & & \\
\hline Únicamente sanidad pública (0-1) & $-19,9$ & $-13,8^{* * *}$ & $-42,0$ & $-6,78^{* * *}$ & $-102,1$ & $-14,2 * * *$ & 1,71 & 0,75 & 67,5 & $19,6 * * *$ & $-2,36$ & $-0,55$ & $-13,8$ & $-2,16 * *$ \\
\hline № de observaciones & \multicolumn{2}{|c|}{15.003} & \multicolumn{12}{|c|}{15.003} \\
\hline seudo $\mathrm{R}^{2}$ & \multirow{2}{*}{\multicolumn{2}{|c|}{$\begin{array}{l}0,1963 \\
-80501\end{array}$}} & \multirow{2}{*}{\multicolumn{12}{|c|}{$\begin{array}{c}0,2595 \\
-17.298,6\end{array}$}} \\
\hline og verosimilitud & & & & & & & & & & & & & & \\
\hline
\end{tabular}

Notas: $\quad * 0,1>$

El Factor de Inflación de la Varianza (FIV) medio de todas las variables independientes es 1,38. Los valores FIV son obtenidos a través de la especificación 1 del Cuadro 3, pero estimando por mínimos cuadrados ordinarios. Dado el elevado número de observaciones (15.003), descartamos la presencia de multicolinealidad en las variables independientes de nuestras 2 especificaciones del Cuadro 3.

Fuente: Elaboración propia a partir de los datos de la EESE 2014.

Revista de Estudios Andaluces, núm. 36 (2018) pp. 178-207. e-ISSN: 2340-2776

http://dx.doi.org/10.12795/rea.2018.i36.08 
24,3\%. Trabajar para el estado emerge por tanto como una opción laboral inclusiva para aquellas personas con algún tipo de limitación, enfermedad o problema de salud.

Las opciones de tener un empleo como asalariado (no funcionario), por el contrario, sí varían en función de las limitaciones para "realizar las actividades que la gente habitualmente hace" (indicadores $a, b$ ). Así, las limitaciones físicas (indicador $a$ ) disminuyen esta probabilidad un 10,7\% mientras que las mentales (indicador $b$ ) lo hacen en torno a un 33\%. Finalmente, tal y como ocurría para toda la muestra en la especificación 1, se observa también para este grupo cómo tener alguna enfermedad o problema de salud físico de gravedad intermedia o moderada (indicador $d$ ) aumenta su probabilidad de trabajar en torno a un $6 \%$. Nuevamente la explicación para este resultado podría ser la diferencia de edad media observada entre las personas con los problemas de salud más leves y el colectivo de personas física y mentalmente sanas, que supera los 4 años para la submuestra de trabajadores asalariados no funcionarios.

Analizamos ahora las probabilidades de encontrarse desempleado. En este caso, las limitaciones físicas (indicador $a$ ) aumentan esta probabilidad sobre un $25 \%$ mientras que las mentales (indicador b) lo hacen un $56,3 \%$. En cuanto a las enfermedades o problemas de salud, observamos cómo no afectan a la probabilidad de desempleo si son de tipo físico (indicadores $c$, $d$ ), mientras que si lo son de tipo mental (indicador $e$ ) aumentan la probabilidad de desempleo un 53,5\%.

La probabilidad de estar inactivo, o bien realizando labores del hogar como actividad principal o bien a través de situaciones de prejubilación, también parece estar afectada por la existencia de limitaciones para realizar actividades cotidianas. En particular, las limitaciones de tipo físico (indicador $a$ ) aumentan la probabilidad de ser inactivo sobre un $14 \%$, mientras que si las limitaciones son de tipo mental (indicador $b$ ) la probabilidad de inactividad aumenta un 42,8\%. Estos resultados son consistentes con el análisis llevado a cabo por Schur y Kruse (2002), en el que observan cómo las personas con discapacidad muestran una mayor probabilidad de dedicarse a la realización de tareas en el hogar. Por su parte, las enfermedades o problemas de salud, independientemente de su tipo o gravedad (indicadores $c-d-e$ ), no parecen afectar a las opciones de inactividad.

En cuanto a las opciones de ser estudiante, estas no se ven afectadas por las limitaciones físicas (indicador $a$ ) mientras que, si las limitaciones son mentales (indicador $b$ ), las opciones de ser estudiante aumentan un $37,5 \%$. Al igual que ocurría para el grupo de inactivos, las enfermedades o problemas de salud, independientemente de su tipo o gravedad (indicadores $c-d-e$ ), no parecen afectar a la probabilidad de ser estudiante.

Finalmente, sería necesaria una reflexión acerca de la relación positiva identificada entre las limitaciones para "realizar las actividades que la gente habitualmente hace" (indicadores $a, b$ ) y la probabilidad de no tener un empleo siendo desempleado, inactivo o estudiante. Indudablemente tener limitaciones para realizar actividades cotidianas debe generar también dificultades para buscar empleo de forma activa, realizar labores del hogar o cursar cualquier tipo de formación reglada. Sin embargo, estas dificultades no suponen riesgo alguno de dejar de pertenecer a estos

Revista de Estudios Andaluces, núm. 36 (2018) pp. 178-207. e-ISSN: 2340-2776 http://dx.doi.org/10.12795/rea.2018.i36.08 
grupos sino tan solo afectan al desempeño individual a la hora de realizar estas tareas. Por el contrario, en perspectiva comparada con las actividades asociadas con tener un empleo (empresario, funcionario o asalariado no funcionario), estas limitaciones no solo dificultan el desempeño potencial sino también las propias opciones de pertenecer a estos colectivos, pues la pertenencia o no se produce en concurrencia competitiva con otros sujetos que no padecen estas limitaciones.

\subsection{RESULTADOS RELATIVOS A LAS VARIABLES DE CONTROL}

Presentamos a continuación algunos resultados relevantes asociados a las variables de control de nuestras especificaciones. En primer lugar, vemos cómo el género juega un papel relevante en la probabilidad de tener un empleo, siendo las mujeres las que presentan en torno a un $22 \%$ menos de probabilidad de tener un empleo que los hombres (especificación 1). Estas menores opciones se concretan en las actividades de empresario y asalariado no funcionario (especificación 2). Así, las mujeres presentan un $66,4 \%$ menos de probabilidad de emprender y en torno a un $16 \%$ menos de probabilidad de ser asalariadas no funcionarias que los hombres. Por el contrario, ser mujer aumenta la probabilidad de ser funcionario en torno a un $10 \%$.

La edad, por su parte, parece favorecer la probabilidad de tener un empleo, si bien el efecto de esta variable parece aumentar a tasa decreciente (el coeficiente asociado a la edad es positivo mientras que el coeficiente asociado a su cuadrado es negativo, siendo ambos coeficientes significativos) (especificación 1). Al desagregar por actividades, este efecto se mantiene en el caso de todas las actividades asociadas a tener un empleo $y$, en consecuencia, se invierte en el caso de algunas actividades asociadas a no tener un empleo, en concreto la inactividad y ser estudiante.

El estado civil también es un determinante importante sobre la actividad laboral y, en concreto, con la probabilidad de tener un empleo. Vemos en este caso cómo, frente a los solteros (nuestra categoría de referencia), los casados, separados y divorciados presentan una mayor probabilidad de tener un empleo, entre un 5 y un 10\% superior (especificación 1). Estos efectos netos, sin embargo, ocultan otros resultados que emergen cuando desagregamos por actividades (especificación 2). Así, los casados y viudos parecen tener una mayor probabilidad de ser empleados asalariados e inactivos y una menor probabilidad de ser desempleados y estudiantes.

En cuanto al número de niños en el hogar, estos parecen disminuir la probabilidad de tener un empleo a razón de un 4\% por niño en el hogar (especificación 1). Esta disminución, sin embargo, afecta sobre todo a la probabilidad de ser asalariado y estudiante (que cada niño disminuye un 6,7\% y un $12,3 \%$ respectivamente). Para los empresarios, funcionarios y desempleados no parece tener efecto alguno, mientras que la probabilidad de ser inactivo aumenta a razón de un $23 \%$ por niño en el hogar.

El nivel educativo, por su parte, también arroja resultados interesantes. Vemos así que un mayor nivel educativo aumenta la probabilidad de tener un empleo (especificación 1), siendo la probabilidad de trabajar de un universitario un 43,5\% superior que la de un individuo con educación básica. Este aumento, sin embargo, surge fundamentalmente de la probabilidad de ser funcionario

Revista de Estudios Andaluces, núm. 36 (2018) pp. 178-207. e-ISSN: 2340-2776

http://dx.doi.org/10.12795/rea.2018.i36.08

Esta obra se distribuye con la licencia Creative Commons Reconocimiento-NoComercial-SinObraDerivada 4.0 Internacional (CC BY-NC-ND 4.0.) 
(especificación 2). Las opciones de acceder a un puesto de funcionario son un $218 \%$ mayor en un universitario que en un individuo con educación básica. En el resto de opciones laborales, esto es, empresario y asalariado, la educación tiene un efecto sustancialmente menor. Por su parte, las probabilidades de ser desempleado e inactivo se reducen un $91 \%$ y un $66 \%$, respectivamente, para personas con estudios universitarios.

Por su parte, los nacidos en España presentan un 8\% mayor de probabilidad de tener un empleo que los nacidos en el extranjero (especificación 1), concentrándose este efecto en las opciones laborales de empresario y funcionario, no así en la de asalariado (especificación 2). En concreto, las opciones de ser empresario aumentan para los nacidos en España un 15\% mientras que las opciones de ser funcionario aumentan un 83,6\%. También observamos cómo los nacidos en España tienen una menor probabilidad de ser desempleados e inactivos, y una mayor probabilidad de ser estudiantes.

El tamaño del municipio parece aumentar las opciones de ser asalariado, mientras que reduce la probabilidad de emprendimiento (especificación 2).

Los resultados relativos a la comunidad autónoma en la que reside el individuo también resultan de interés. La probabilidad de tener un empleo es mayor en todas las comunidades y ciudades autónomas que en Andalucía (nuestra categoría de referencia), con la excepción de Extremadura y Melilla. En particular, las comunidades que presentan una mayor probabilidad de tener un empleo son Islas Baleares, Navarra, Madrid, Aragón, La Rioja, País Vasco, Cataluña, y Castilla y León, todas ellas con una probabilidad entre un 18 y un $24 \%$ mayor que en Andalucía (especificación 1 ).

Al distinguir por actividades (especificación 2), vemos cómo las mayores opciones de emprendimiento las encontramos en Galicia, Castilla y León, Asturias y Cantabria, con un 48\%, un $38 \%$, un $28 \%$ y un $25 \%$ más de probabilidad que Andalucía, respectivamente. Por el contrario, la menor probabilidad se detecta en Ceuta (un $46 \%$ menos que en Andalucía).

A la hora de ser funcionario, y en comparación con Andalucía, esta probabilidad es más de un $130 \%$ mayor en Ceuta y Melilla, un $89 \%$ mayor en Extremadura, un 51,5\% mayor en Castilla - La Mancha, en torno a un $40 \%$ mayor en Castilla y León, La Rioja y Asturias. Las menores probabilidades las encontramos en Cataluña, Madrid y el País Vasco, con un $44 \%$, un $40 \%$ y un $36 \%$ menos que en Andalucía, respectivamente.

En cuanto a la probabilidad de ser asalariado no funcionario, las mayores probabilidades se detectan en Madrid, País Vasco, Cataluña, Islas Baleares, Navarra y Aragón, siendo esta probabilidad en todos estos casos entre un $30 \%$ y un $42 \%$ mayor que en Andalucía. La menor probabilidad en ese caso surge en Melilla, siendo la opción de empleo asalariado un 18\% menor que en Andalucía.

Finalmente, la variable económica también arroja resultados relevantes. Así, aquellos individuos cuya modalidad de seguro sanitario se limita a la sanidad pública presentan un $20 \%$ menor de probabilidad de tener un empleo (especificación 1). Esta menor probabilidad se concreta por

Revista de Estudios Andaluces, núm. 36 (2018) pp.178-207. e-ISSN: 2340-2776 http://dx.doi.org/10.12795/rea.2018.i36.08

(c) (i) $\ominus$

cc) Esta obra se distribuye con la licencia Creative Commons Reconocimiento-NoComercial-SinObraDerivada 4.0 Internacional (CC BY-NC-ND 4.0.) 
actividades en una menor probabilidad de ser empresario (un $42 \%$ menos), funcionario (un $102 \%$ menos) y estudiante (un $14 \%$ menos) y en una mayor probabilidad de ser desempleado (un $67,5 \%$ más) (especificación 2).

\section{CONCLUSIONES}

A pesar de los enormes cambios sufridos por el mercado laboral español en los últimos años, la situación de las personas con discapacidad, en términos de integración laboral, sigue caracterizándose por unas tasas de actividad y empleo alarmantemente menores y unas cifras de desempleo claramente superiores a las que presentan las personas sin discapacidad. Sin embargo, los estudios para el caso español que relacionan discapacidad y actividad económica son escasos y analizan principalmente la participación laboral, esto es, la mera pertenencia a la población activa. Utilizando los microdatos de la EESE 2014, este trabajo presenta un análisis condicional que permite analizar el papel de diferentes tipos de limitaciones, enfermedades y problemas de salud en relación con la probabilidad de tener un empleo en España.

Sobre la base de los resultados obtenidos, no parece que el colectivo de personas con limitaciones, enfermedades o problemas de salud pueda ser considerado como un grupo homogéneo. Por el contrario, si bien las limitaciones de tipo físico disminuyen la probabilidad de tener un empleo, son las personas con algún tipo de limitación, enfermedad o problema de salud mental las que presentan unas menores opciones de emplearse. Estos resultados tienen implicaciones para el diseño de las políticas sociales y de empleo dirigidas a este colectivo más vulnerable. En particular, se hace patente la necesidad de reforzar aquellas medidas activas y preventivas dirigidas a estos grupos con mayor riesgo de exclusión.

Como una de sus contribuciones principales, este análisis permite también identificar la relación entre los diferentes tipos de limitaciones, enfermedades y problemas de salud, y las probabilidades de llevar a cabo diferentes actividades principales: empresario, funcionario, asalariado no funcionario, desempleado, inactivo y estudiante. Así, mientras las limitaciones físicas y mentales limitan las opciones de tener un empleo como empresario o asalariado no funcionario, por el contrario, ninguna de estas limitaciones tiene influencia alguna sobre la probabilidad de ser funcionario. Es de justicia reconocer, por tanto, el papel que está ejerciendo el estado en la inclusión laboral de las personas con discapacidad en España.

Por su parte, en cuanto a las actividades principales asociadas a situaciones en las que el individuo no tiene un empleo, esto es, desempleo, inactividad y estudiante, estas son más frecuentes en presencia de limitaciones, ya sean físicas o mentales. Estos resultados son coherentes, no solo con las elevadas cifras agregadas de desempleo para este colectivo, que emergen a consecuencia de una escasez de demanda de trabajo, sino también con sus bajas tasas de actividad, en los que la escasez de oferta de trabajo es la responsable. En aras de promover la inclusión laboral del mismo, parece necesario reforzar tanto aquellas políticas de demanda como de oferta. $\mathrm{O}$ dicho de otro modo, y tal y como sugieren algunos trabajos previos para España (ej. Malo 2001, 2003, 2004; Dávila 2006, Cueto et al. 2012), no parece suficiente con una política socio-laboral que persiga fomentar las transiciones del desempleo al empleo, sino que también siga promoviendo las transiciones hacia la actividad.

Revista de Estudios Andaluces, núm. 36 (2018) pp. 178-207. e-ISSN: 2340-2776

http://dx.doi.org/10.12795/rea.2018.i36.08

Esta obra se distribuye con la licencia Creative Commons Reconocimiento-NoComercial-SinObraDerivada 4.0 Internacional (CC BY-NC-ND 4.0.) 


\section{AGRADECIMIENTOS}

Queremos expresar nuestra gratitud a Begoña Cueto (Universidad de Oviedo), Fernando Millán (Universidad de Sevilla), Concepción Román (Universidad de Huelva), Juan Alberto Sanchis (Universidad de Valencia) y a los evaluadores anónimos por sus comentarios y sugerencias. Este trabajo se ha beneficiado de la ayuda financiera recibida del Grupo de Investigación SEJ-487 (Spanish Entrepreneurship Research Group - SERG), de la Estrategia de Política de Investigación y Transferencia 2018 de la Universidad de Huelva, y del Ministerio de Economía, Industria y Competitividad (Proyecto ECO2017-86402-C2-2-R).

\section{REFERENCIAS}

Acemoglu, D., Angrist, J.D. (2001). Consequence of employment protection? The case of the Americans with Disabilities Act. Journal of Political Economy 19(5), pp. 915-950. doi: https://doi.org/10.1086/322836

Albarrán, I., Alonso, P. (2010). Participación en el mercado laboral español de las personas con discapacidad y en situación de dependencia. Papeles de Población 16(64), pp. 217-256.

Baldwin, M., Johnson, W.G. (1994). Labor market discrimination against men with disabilities. Journal of Human Resources 29(1), pp. 1-19. doi: https://doi.org/10.2307/146053

Baldwin, M., Johnson, W.G. (1995). Labor market discrimination against women with disabilities. Industrial Relations 34(4), pp. 555-571. doi: https://doi.org/10.1111/j.1468-232X.1995.tb00388.x

Baldwin, M., Johnson, W.G. (2000). Labor market discrimination against men with disabilities in the year of the ADA. Southern Economic Journal 66(3), pp. 548-566. doi: https://doi.org/10.2307/1061425

Benítez-Silva, H., Buchinsky, M., Chan, H.M., Rust, J., Sheidvasser, S. (1999). An empirical analysis of the social security disability application, appeal, and award process. Labour Economics 6(2), pp. 147178. doi: https://doi.org/10.1016/S0927-5371(99)00014-7

Berthoud, R. (2003). Disabled people and jobs. Benefits 11(3), pp. 163-168.

Blackaby, D., Clark, K., Drinkwater, S., Leslie, D., Murphy, P., O'Leary, N. (1999). Earnings and employment opportunities of disabled people. Research Report No. 133, Department for Education and Employment, Nottingham.

Revista de Estudios Andaluces, núm. 36 (2018) pp.178-207. e-ISSN: 2340-2776 http://dx.doi.org/10.12795/rea.2018.i36.08

(c) $($ ) $(\ominus)$

cc) Esta obra se distribuye con la licencia Creative Commons Reconocimiento-NoComercial-SinObraDerivada 4.0 Internacional (CC BY-NC-ND 4.0.) 
Blanck, P.D., Sandler, L.A., Schmeling, J.L., Schwartz, H.A. (2000). The emerging workforce of entrepreneurs with disabilities: preliminary study of entrepreneurship in lowa. lowa Law Review 85, pp. 1583-1668.

Boylan, A., Burchardt, T. (2002). Barriers to self-employment for disabled people. Report for the Small Business Service, HMSO, London.

Cantanero, D., Moreno, P. (2010). Dependencia y empleo: un análisis empírico con la Encuesta de Discapacidades y Atención a la Dependencia (EDAD) 2008. Serie Documentos de Trabajo 578/2010, Fundación de las Cajas de Ahorro

Casado, D. (2004). Conocimiento y gestión del empleo de las personas con discapacidad. Revista del Ministerio de Trabajo y Asuntos Sociales 50, pp. 47-72.

Cazallas, C. (2005). La desigualdad en el mercado de trabajo de personas con discapacidad. Revista Universitaria de Ciencias del Trabajo 6, pp. 147-164.

Cortés Ortiz, M. (2011). La Responsabilidad Social Empresarial en el ámbito de la discapacidad (RSED). Dimensión, contenido y tendencias en época de crisis. Colección Barclays Diversidad e Inclusión, número 5.

Cueto, B., Malo, M.A., Rodríguez, V. (2012). La brecha de participación laboral de las personas con discapacidad en España. Un análisis con la EDAD-2008. Cuadernos del Mercado de Trabajo 8, pp. 20-27.

Dávila, C.D. (2006). Discapacidad y género. Un estudio de participación en el mercado de trabajo español. Moneda y Crédito 223, pp. 127-158.

Dávila, C.D., Malo, M.A. (2006). Género, discapacidad y posición familiar. La participación laboral de las mujeres con discapacidad. Cuadernos Aragoneses de Economía 16(1), pp. 61-82.

DeLeire, T. (2000). The wage and employment effects of the Americans with Disabilities Act. Journal of Human Resources 35(4), pp. 693-715. doi: https://doi.org/10.2307/146368

Disney, R., Emmerson, C., Wakefield, M. (2006). III health and retirement in Britain: a panel data based analysis. Journal of Health Economics 25(4), pp. 621-649. doi: https://doi.org/10.1016/j.jhealeco.2005.05.004

Gannon, B. (2005). A dynamic analysis of disability and labour force participation. Health Economics 14(9), pp. 925-938. doi: https://doi.org/10.1002/hec.1044

Gannon, B., Nolan, B. (2007). Transitions in disability and work. Estudios de Economía Aplicada 25(2), pp. 447-472. 
García Mainar, I. (2000). Estado de salud, situación laboral y salarios en España. Cuadernos Aragoneses de Economía 10(1), pp. 233-245.

Giménez, D., Ramos, M. (2003). La discriminación de las mujeres discapacitadas en España. Revista del Ministerio de Trabajo y Asuntos Sociales 45, pp. 61-76.

Gudex, C., Lafortune, G. (2000). An Inventory of Health and Disability-Related Surveys in OECD Countries. Labour Market and Social Policy Occasional Papers 44, OCDE, París. doi: https://doi.org/10.1787/337271133800

Hale, T., Hayghe, H., McNeil, J. (1998). Persons with disabilities: Labor market activity, 1994. Monthly Labor Review 121(9), pp. 3-12.

Hernández, J., Millán, J.M. (2015). Las personas con discapacidad en España: Inserción laboral y crisis económica. Revista Española de Discapacidad 3(1), pp. 29-56. doi: https://doi.org/10.5569/23405104.03.01.02

Hotchkiss, J.L. (2004). Growing part-time employment among workers with disabilities: marginalization or opportunity? Economic Review Federal Reserve Bank of Atlanta, Atlanta, GA, third quarter.

Hum, D., Simpson, W. (1996). Canadians with disabilities and the labour market. Canadian Public Policy 22(3), pp. 285-299. doi: https://doi.org/10.2307/3551506

Jenkins, S., Rigg, J. (2004). Disability and disadvantage: Selection, onset, and duration effects. Journal of Social Policy 33(3), pp. 479-501. doi: https://doi.org/10.1017/S0047279404007780

Jiménez, A., Huete, A. (2010). Estadísticas y otros registros sobre discapacidad en España. Política y Sociedad 47(1), pp. 165-173.

Johnson, W.G., Lambrinos, J. (1985). Wage discrimination against handicapped men and women. Journal of Human Resources 20(2), pp. 264-277. doi: https://doi.org/10.2307/146012 Jones, M.K. (2006). Is there employment discrimination against the disabled? Economics Letters 92(1), pp. 32-37. doi: https://doi.org/10.1016/j.econlet.2006.01.008

Jones, M.K. (2008). Disability and the labour market: a review of the empirical evidence. Journal of Economic Studies 35(5), pp. 405-424. doi: https://doi.org/10.1108/01443580810903554

Jones, M.K. (2011). Disability, employment and earnings: an examination of heterogeneity. Applied Economics 43(8), pp. 1001-1017. doi: https://doi.org/10.1080/00036840802600053

Jones, M.K., Latreille, P.L., Sloane, P.J. (2006). Disability, gender and the British labour market. Oxford Economic Papers 58(3), pp. 407-459. doi: https://doi.org/10.1093/oep/gpl004

Revista de Estudios Andaluces, núm. 36 (2018) pp.178-207. e-ISSN: 2340-2776 http://dx.doi.org/10.12795/rea.2018.i36.08

(c) (i) $\ominus$

cC. EY NG ND Esta obra se distribuye con la licencia Creative Commons Reconocimiento-NoComercial-SinObraDerivada 4.0 Internacional (CC BY-NC-ND 4.0.) 
Kidd, M.P., Sloane, P.J., Ferko, I. (2000). Disability and the labour market: an analysis of British males. Journal of Health Economics 19(6), pp. 961-981. doi: https://doi.org/10.1016/S01676296(00)00043-6

Kreider, B., Pepper, J.V. (2007). Disability and employment. Reevaluating the evidence in light of reporting errors. Journal of the American Statistical Association 102(478), pp. 432-441. doi: https://doi.org/10.1198/016214506000000997

Kreider, B., Pepper, J.V. (2008). Inferring disability status from corrupt data. Journal of the Applied Econometrics 23(3), pp. 329-349. doi: https://doi.org/10.1002/jae.979

Kruse, D., Schur, L. (2003). Employment of people with disabilities following the ADA. Industrial Relations 42(1), pp. 31-64. doi: https://doi.org/10.1111/1468-232X.00275

Lalive, R., Wuellrich, J., Zweimuller, J. (2013). Do financial incentives affect firms' demand for disabled workers? Journal of the European Economic Association 11(1), pp. 25-58. doi: https://doi.org/10.1111/j.1542-4774.2012.01109.x

Livermore, G.A., Stapleton, D.C., Nowak, M.W., Wittenburg, D.C., Eiseman, E.D. (2000). The Economics of Policies and Programs affecting the Employment of People with Disabilities. Cornell University. Ithaca, NY. doi: https://doi.org/10.2139/ssrn.892439

López, C.M., Seco, E. (2005). Discapacidad y empleo en España: su visibilidad. INNOVAR. Revista de Ciencias Administrativas y Sociales 15(26), pp. 59-72.

Loprest, P., K. Ru, S.H. Sandell (1995). Gender, disabilities, and employment in the health and retirement survey. Journal of Human Resources 30, pp. S293-S314. doi: https://doi.org/10.2307/146286

Madden, D. (2004). Labour market discrimination on the basis of health: an application to UK data. Applied Economics 36(5), pp. 421-442. doi: https://doi.org/10.1080/00036840410001682133

Malo, M.A. (2001). Discapacidad y participación en el mercado de trabajo. En: Garrido, L. y Toharia, L. (Eds.), Condiciones de vida en España y en Europa (pp.281-306). Madrid, España: Instituto Nacional de Estadística.

Malo, M.A. (2003). Las personas con discapacidad en el mercado de trabajo español. Revista del Ministerio de Trabajo y Asuntos Sociales 46, pp. 99-126.

Malo, M.A. (2004). ¿Cómo afectan las discapacidades a la probabilidad de ser activo en España? Un análisis empírico con datos de la Encuesta sobre Discapacidades, Deficiencias y Estado de la Salud de 1999. Cuadernos de Economía 27, pp. 75-108. 
Malo, M.A. (2007). La definición de la discapacidad en la investigación económica: una reflexión necesaria sobre qué características debería cumplir. Estudios de Economía Aplicada 25(2), pp. 407428.

Malo, M.A., Cueto, B., Rodríguez, V. (2011). Compatibilidad entre pensiones contributivas por incapacidad y empleo: el caso español. Cuadernos de Relaciones Laborales 29(1), pp. 125-153. doi: https://doi.org/10.5209/rev_CRLA.2011.v29.n1.5

Malo, M.A., Pagán, R. (2005). Participación laboral y discapacidad: el caso de la Comunidad Andaluza. Revista de Estudios Regionales 74, pp. 89-116.

Pagán, R., Marchante, A.J. (2004). Mercado laboral de las personas discapacitadas en España. Trabajo - Revista Andaluza de Relaciones Laborales 13, pp. 207-226.

Parsons, D. (1982). The male labor force participation decision: Health, reported health, and economic incentives. Economica 49(193), pp. 81-91. doi: https://doi.org/10.2307/2553527

Reeves, A., Karanikolos, M., Mackenbach, J., McKee, M., Stuckler, D. (2014). Do employment protection policies reduce the relative disadvantage in the labour market experienced by unhealthy people? A natural experiment created by the Great Recession in Europe. Social Science \& Medicine 121, pp. 98-108. doi: https://doi.org/10.1016/j.socscimed.2014.09.034

Riphahn, R. (1999). Income and employment effects of health shocks. A test case for the German welfare state. Journal of Population Economics 12(3), pp. 363-389. doi: https://doi.org/10.1007/s001480050104

Rodríguez-Cabrero, G., C. García-Serrano, L. Toharia (2009). Evaluación de las políticas de empleo para personas con discapacidad y formulación y coste económico de nuevas propuestas de integración laboral. Colección Telefónica Accesible 9, Telefónica-CERMI- Madrid, España: Ed. Cinca.

Rodríguez, V. (2012a). Las políticas de empleo orientadas a personas con discapacidad: ¿evolución o retroceso? Revista del Ministerio de Empleo y Seguridad Social 101, pp. 77-96.

Rodríguez, V. (2012b). El empleo de las personas con discapacidad en la Gran Recesión: ¿son los Centros Especiales de Empleo una excepción? Estudios de Economía Aplicada 30(1), pp. 237-259.

Rodríguez, V. (2013). Fuentes de información sobre discapacidad y empleo en España. Revista Española de Discapacidad 1(1), pp. 73-95. doi: https://doi.org/10.5569/2340-5104.01.01.04

Rodríguez, V. (2017). El empleo de las personas con discapacidad en España: quo venis, quo vadis. Panorama Social 26, pp. 41-55. 
Rodríguez, V., Cueto, B. (2013). El trabajo de las personas con discapacidad ante la crisis. Revista Internacional de Organizaciones 11, pp. 61-86. doi: https://doi.org/10.17345/rio11.61-86

Ros-Clemente, M.I. (2017). Estudio de la inclusión laboral de las personas con discapacidad en España. Tesis Doctoral - Universidad Católica de Murcia.

Schur, L. (2003). Barriers or opportunities? The causes of contingent and part-time work among people with disabilities. Industrial Relations 42(4), pp. 589-622. doi: https://doi.org/10.1111/1468232X.00308

Schur, L., D. Kruse (2002). Non-standard work arrangements and disability income. Report to the Disability Research Institute, University of Illinois Urbana-Champaign, Champaign, IL, August.

Stern, S. (1989). Measuring the effect of disability on labor force participation. Journal of Human Resources 24(3), pp. 361-395. doi: https://doi.org/10.2307/145819

Villa-Fernández, N. (2003). Situación laboral de las personas con discapacidad en España. Revista Complutense de Educación 14(2), pp. 393-424.

Wilkins, R. (2004). The effects of disability on labour force status in Australia. Australian Economic Review 37(4), pp. 359-382. doi: https://doi.org/10.1111/j.1467-8462.2004.00338.x

Zwerling, C., P.S. Whitten, N.L. Sprince, C.S. Davis, R.B. Wallace, P. Blanck, S.G. Herringa (2002). Workforce participation by persons with disabilities: The National Health Interview Survey Disability Supplement. Journal of Occupational and Environmental Medicine 44(4), pp. 358-364. doi: https://doi.org/10.1097/00043764-200204000-00017

Yelin, E., P. Katz (1994). Labor force trends among persons with and without disabilities. Monthly Labour Review 117(10), pp. 36-42. 


\section{APÉNDICE}

\section{Cuadro A1: Descripción detallada de las variables utilizadas en el análisis.}

Variable

Especificación 1

Tener un empleo (0-1)

Especificación 2

Actividad Principal (1-6)

\section{Variables principales}

Estado de salud (6 variables ficticias)

Descripción

\section{Variables dependientes}

Variable discreta binaria que toma el valor 1 para individuos que trabajan como empresario con o $\sin$ asalariados $(F 13=5,6)$, funcionario $(F 13=1)$ o trabajador con contrato indefinido o temporal $(F 13=2,3)$. La variable toma el valor 0 para individuos que no trabajan, y se encuentran en alguna de las siguientes situaciones: desempleado $\left(A 11_{-} i=2\right)$, realizando labores del hogar $\left(A 11_{-} i=6\right)$, prejubilado $\left(A 11_{-} i=3\right)$ o estudiante (A11_i = 4).

Variable discreta no ordenada que toma el valor 1 para individuos que trabajan como empresario con o sin asalariados $(F 13=5,6)$, el valor 2 para individuos que trabajan como funcionario (F13 = 1), el valor 3 para individuos que trabajan con contrato indefinido o temporal $(F 13=2,3)$, el valor 4 para individuos desempleados (A11_i $=2)$, el valor 5 para individuos inactivos (prejubilados o realizando labores del hogar; $\left(\bar{A} 11_{-} i=3,6\right)$ y el valor 6 para estudiantes $\left(\mathrm{A} 11 \_\mathrm{i}=4\right)$.

\section{Variables independientes}

a. Limitación física (0-1): Variable ficticia que toma el valor 1 para individuos que, durante al menos los últimos 6 meses, se han visto limitados o gravemente limitados físicamente para realizar las actividades que la gente habitualmente hace debido a un problema de salud (G23 = 1-2 + G24 = 1)

b. Limitación mental -o física y mental- (0-1): Variable ficticia que toma el valor 1 para individuos que, durante al menos los últimos 6 meses, se han visto limitados o gravemente limitados mentalmente (o física y mentalmente) para realizar las actividades que la gente habitualmente hace debido a un problema de salud (G23 = 1-2 + G24 = 2-3). c. Enfermedad o problema de salud físico severo (0-1): Variable ficticia que toma el valor 1 para individuos que, no habiéndose visto limitados o gravemente limitados física y/o mentalmente para realizar las actividades que la gente habitualmente hace durante al menos los últimos 6 meses, sí han padecido en los últimos 12 meses al menos uno de los siguientes problemas de salud o enfermedades: infarto de miocardio (G25b_2 = 1); angina de pecho, enfermedad coronaria (G25b_3 = 3); otras enfermedades del corazón (G25b_4 = 1); bronquitis crónica, enfisema, enfermedad pulmonar obstructiva crónica (G25b_11 = 1); úlcera de estómago o duodeno (G25b_13 = 1); cirrosis, disfunción hepática (G25b_19=1); ictus (embolia, infarto cerebral, hemorragia cerebral) (G25b_23 $=1$ ); tumores malignos (G25b_26=1); lesiones o defectos permanentes causados por un accidente (G25b_32 $=1$ ).

d. Enfermedad o problema de salud físico de gravedad intermedia o moderada (0-1): Variable ficticia que toma el valor 1 para individuos que, no habiéndose visto limitados o gravemente limitados física y/o mentalmente para realizar las actividades que la gente habitualmente hace durante al menos los últimos 6 meses, sí han padecido en los últimos 12 meses al menos uno de los siguientes problemas de salud o enfermedades: tensión alta (G25b_1 = 1); varices en las piernas (G25b $5=1$ ); artrosis (excluyendo artritis) (G25b_6 = 1); dolor de espalda crónico (cervical) (G25b_7 = 1); dolor de espalda crónico (lumbar) (G25b_8 =1); alergia crónica, como rinitis, conjuntivitis o dermatitis alérgica, alergia alimentaria o de otro tipo (asma alérgica excluida) $\left(G 25 b \_9=1\right)$; asma (incluida asma alérgica) (G25b_10 =1), diabetes (G25b_12 $=1$ ); incontinencia urinaria o problemas de control de la orina (G25b_14 = 1); colesterol alto (G25b_15 = 1); cataratas (G25b_16 =1); problemas crónicos de piel $\left(G 25 b \_17=1\right)$; estreñimiento crónico (G25b_18 = 1); diabetes (G25b_12 = 1); migraña o dolor de cabeza frecuente $\left(G 25 b \_24=1\right)$; hemorroides $\left(G 25 b \_25=1\right)$; osteoporosis $\left(G 25 b \_27=1\right)$; Problemas de tiroides

Revista de Estudios Andaluces, núm. 36 (2018) pp.178-207. e-ISSN: 2340-2776

http://dx.doi.org/10.12795/rea.2018.i36.08

c) (i) $\odot \ominus$

Esta obra se distribuye con la licencia Creative Commons Reconocimiento-NoComercial-SinObraDerivada 4.0 Internacional (CC BY-NC-ND 4.0.) 
$\left(G 25 b \_28=1\right)$, problemas de riñón $\left(G 25 b \_29=1\right)$, problemas de próstata -solo hombres- $\left(\mathrm{G} 25 \mathrm{~b} \_30=1\right)$; problemas debidos al periodo menopaúsico -solo mujeres$\left(\mathrm{G} 25 \mathrm{~b} \_31=1\right)$.

e. Enfermedad o problema de salud mental -o físico y mental- (0-1): Variable ficticia que toma el valor 1 para individuos que, no habiéndose visto limitados o gravemente limitados física y/o mentalmente para realizar las actividades que la gente habitualmente hace durante al menos los últimos 6 meses, sí han padecido en los últimos 12 meses al menos uno de los siguientes problemas de salud mental o enfermedades mentales: depresión $\left(G 25 b \_20=1\right)$, ansiedad crónica $\left(G 25 b \_21=3\right)$ u otros problemas mentales $\left(G 25 b \_22=1\right)$, pudiendo además haber padecido una enfermedad o problema de salud físico, independientemente de su gravedad (G25b_1-19=1, G25b_23-32 =1).

$f$. Individuo física y mentalmente sano (0-1) (ref.): Variable ficticia que toma el valor 1 para individuos que, durante al menos los últimos 6 meses, no se han visto limitados ni física ni mentalmente para realizar las actividades que la gente habitualmente hace debido a un problema de salud $(G 23=3+G 24=3)$, y además no han padecido en los últimos 12 meses ninguna enfermedad o problema de salud de tipo físico ni mental (G25b_1-32 =0).

\section{Variables de control}

Variables demográficas

Mujer (0-1)

Edad (18-65)

Estado civil (5 variables ficticias)

Número de niños en el hogar (0-6)

Nivel de estudios (4 variables ficticias)

Variables geográficas

Nacido en España (0-1)

Tamaño del municipio (1-7)
Variable ficticia que toma el valor 1 para mujeres (SEXOa $=1$ ).

Edad del individuo, variando entre 18 y 65 años (EDADa).

5 variables ficticias que toman el valor 1 para aquellos individuos cuyo estado final sea el siguiente: Soltero (ref.) $(E 4 b=1)$, Casado $(E 4 b=2)$, Separado $(E 4 b=4)$, Divorciado (E4b $=5)$, y Viudo $(E 4 b=3)$.

Número de niños menores de 15 años que viven en el hogar. Esta variable varía entre 0 y 6 (NMENORES).

Educación básica (0-1) (ref.): Variable ficticia que toma el valor 1 para individuos que no saben leer o escribir (ESTUDIOS $=2$ ), que no han completado la educación primaria (ESTUDIOS = 3) o que sí han completado la educación primaria, pero no han completado la primera etapa de la enseñanza secundaria $($ ESTUDIOS $=4)$.

Educación secundaria (0-1): Variable ficticia que toma el valor 1 para individuos que sí han completado la primera etapa de la enseñanza secundaria (ESTUDIOS $=5$ ) o han completado los estudios de bachillerato (ESTUDIOS $=6$ ).

Educación profesional (0-1): Variable ficticia que toma el valor 1 para individuos que han completado enseñanzas profesionales de grado medio o equivalentes $($ ESTUDIOS $=7$ ) 0 de grado superior o equivalentes (ESTUDIOS $=8$ ).

Educación universitaria (0-1): Variable ficticia que toma el valor 1 para individuos que han completado estudios universitarios o equivalentes (ESTUDIOS $=9$ ).

Variable ficticia que toma el valor 1 para individuos nacidos en España $\left(E 1 \_1=1\right)$.

Variable ordenada discreta que toma valores entre 1 y 7 y que captura el tamaño del municipio de residencia. La variable toma el valor 1 para municipios con menos de 10.000 habitantes (ESTRATO $=6$ ), el valor 2 para municipios de 10.000 a 20.000 habitantes (ESTRATO = 5), el valor 3 para municipios de 20.000 a 50.000 habitantes (ESTRATO $=4$ ), el valor 4 para municipios de 50.000 a 100.000 habitantes (ESTRATO $=3$ ), el valor 5 para municipios de 100.000 a 500.000 habitantes que no sean capitales de provincia (ESTRATO =2), el valor 6 para municipios capitales de provincia que tengan menos de 500.000 habitantes (ESTRATO $=1$ ) y el valor 7 para todos los municipios con más de 500.000 habitantes (ESTRATO $=0$ ).

Revista de Estudios Andaluces, núm. 36 (2018) pp. 178-207. e-ISSN: 2340-2776

http://dx.doi.org/10.12795/rea.2018.i36.08 
Comunidad (o ciudad) autónoma (19 variables ficticias)

Variables económicas

Únicamente sanidad pública (0-1)
19 variables ficticias que toman el valor 1 para aquellos individuos que viven en la correspondiente comunidad (o ciudad autónoma) española: Andalucía (ref.) (CCAA =1), Aragón (CCAA = 2), Asturias (CCAA = 3), Islas Baleares (CCAA $=4)$, Islas Canarias (CCAA = 5), Cantabria (CCAA = 6), Castilla y León (CCAA = 7), Castilla - La Mancha (CCAA = 8), Cataluña (CCAA = 9), Comunidad Valenciana $(C C A A=10)$, Extremadura (CCAA $=11)$, Galicia (CCAA = 12), Madrid (CCAA = 13), Murcia (CCAA = 14), Navarra (CCAA = 15), País Vasco $($ CCAA = 16), La Rioja (CCAA = 17), Ceuta $($ CCAA = 18) o Melilla $(C C A A=19)$.

Variable ficticia que toma el valor 1 para individuos cuya modalidad de seguro médico sea únicamente la sanidad pública, sin que el individuo esté asegurado a través de alguna de las mutualidades del estado $\left(084 \_1=1\right)$ y el valor 0 para individuos asegurados a través de estas mutualidades en la sanidad pública $\left(084 \_2=1\right)$ o privada $\left(084 \_3=1\right)$, o a través de la sanidad privada, mediante concierto individual $\left(084 \_4=1\right)$ o a través de la empresa en que trabajen $\left(084 \_5=1\right)$.

Fuente: EESE 2014.

Revista de Estudios Andaluces, núm. 36 (2018) pp.178-207. e-ISSN: 2340-2776 http://dx.doi.org/10.12795/rea.2018.i36.08 